\title{
Probing dark matter freeze-in with long-lived particle signatures: MATHUSLA, HL-LHC and FCC-hh
}

\author{
J.M. No, ${ }^{a, c}$ P. Tunney ${ }^{b, c}$ and B. Zaldivar ${ }^{a}$ \\ ${ }^{a}$ Departamento de Fisica Teorica and Instituto de Fisica Teorica, \\ IFT-UAM/CSIC, Cantoblanco, 28049, Madrid, Spain \\ ${ }^{b}$ Institute for Theoretical Particle Physics and Cosmology (TTK), RWTH Aachen University, \\ D-52056 Aachen, Germany \\ ${ }^{c}$ Theoretical Particle Physics and Cosmology, \\ Department of Physics, King's College London, London WC2R 2LS, U.K. \\ E-mail: Josemiguel.no@uam.es, tunney@physik.rwth-aachen.de, \\ bryan.zaldivarm@uam.es
}

ABSTRACT: Collider searches for long-lived particles yield a promising avenue to probe the freeze-in production of Dark Matter via the decay of a parent particle. We analyze the prospects of probing the parameter space of Dark Matter freeze-in from the decay of neutral parent particles at the LHC and beyond, taking as a case study a freeze-in Dark Matter scenario via the Standard Model Higgs. We obtain the projected sensitivity of the proposed MATHUSLA surface detector (for MATHUSLA100 and MATHUSLA200 configurations) for long-lived particle searches to the freeze-in Dark Matter parameter space, and study its complementarity to searches by ATLAS and CMS at HL-LHC, as well as the interplay with constraints from Cosmology: Big-Bang Nucleosynthesis and Lyman- $\alpha$ forest observations. We then analyze the improvement in sensitivity that would come from a forward detector within a future $100 \mathrm{TeV} p p$-collider. In addition, we discuss several technical aspects of the present Dark Matter freeze-in scenario: the role of the electroweak phase transition; the inclusion of thermal masses, which have been previously disregarded in freeze-in from decay studies; the impact of $2 \rightarrow 2$ scattering processes on the Dark Matter relic abundance; and the interplay between freeze-in and super-WIMP Dark Matter production mechanisms.

Keywords: Beyond Standard Model, Cosmology of Theories beyond the SM, Higgs Physics

ARXIV EPRINT: 1908.11387 


\section{Contents}

1 Introduction 1

2 Dark Matter through the freeze-in mechanism: review 3

3 Freeze-in (from decay) via the Standard Model Higgs 5

3.1 The model 5

3.2 DM relic density: the role of EW symmetry breaking \& thermal masses $\quad 7$

3.3 Super-WIMP contribution to the DM relic abundance 11

4 Constraints on Dark Matter freeze-in from cosmology 12

5 Probing freeze-in Dark Matter with the MATHUSLA detector 14

6 Searches for freeze-in Dark Matter with ATLAS and CMS 17

6.1 Standard Dark Matter searches: $E_{T}^{\text {miss }}($ mono- $X$ ) signatures 17

$\begin{array}{lll}6.2 & \text { Disappearing track signatures } & 19\end{array}$

$\begin{array}{lll}6.3 & \text { Displaced Vertex (DV) signatures } & 19\end{array}$

7 Freeze-in at a $\sqrt{s}=100 \mathrm{TeV}$ collider with a forward detector 21

8 Conclusions 23

A $1 \rightarrow 2$ vs. $2 \rightarrow 2$ freeze-in processes $\quad 24$

B $13 \mathrm{TeV}$ ATLAS DV $+E_{T}^{\text {miss }}$ search recast $\quad 27$

C Higgsino pair production cross sections at $\sqrt{s}=13,100 \mathrm{TeV} \quad 29$

\section{Introduction}

While thermal freeze-out has long been considered the paradigm for Dark Matter (DM) production in the early Universe, experimental efforts over the last 30 years have found no conclusive evidence of the existence of this type of DM. At the same time, searches for DM in direct detection experiments $[1,2]$, via indirect detection $[3,4]$ and through collider probes (e.g. at the LHC $[5,6]$ ) yield at present strong bounds on the interaction strength between DM and the Standard Model (SM) particles.

This provides motivation to consider alternative non-thermal DM production mechanisms in the early Universe. Among these, the freeze-in mechanism [7, 8] (see [9] for a recent review) constitutes a very appealing possibility for its simplicity and the fact that it can be invoked in a wide range of well-motivated extensions of the SM (see e.g. [10-21]). 
In freeze-in scenarios, DM particles are very feebly coupled to the thermal bath in the early Universe and never achieve thermal equilibrium, yet the coupling between DM and the thermal bath particles allows DM to be produced in decays and/or scatterings of bath particles. ${ }^{1}$ Through these processes, the DM abundance slowly increases towards equilibrium, however without ever reaching it. For renormalizable interactions between DM and the thermal bath, the production of DM is most efficient for temperatures $T$ around $\max \left(m_{A}, m_{\mathrm{DM}}\right), m_{A}$ being the mass of the particle $A$ whose scatterings/decays produce DM. ${ }^{2}$ These processes shut-off (DM "freezes-in") soon after $T$ drops below $m_{A}$ or $m_{\mathrm{DM}}$.

Given the feeble interactions between DM and the visible sector, freeze-in DM candidates are naturally compatible with the current null results from DM experimental searches, and probing these scenarios may be more challenging than that of thermal DM candidates. ${ }^{3}$ Nevertheless, when freeze-in DM production in the early Universe proceeds via the decay of thermal bath particles [8], the corresponding feeble coupling would make these particles long-lived. Searches for long-lived particles (LLPs) at the LHC and beyond (see [27, 28]) have received renewed attention in recent years from the lack of conclusive signals in prompt LHC searches for physics beyond the SM, and provide a promising avenue for probing freeze-in DM scenarios [29-34].

In this work we analyze the prospects of probing the parameter space of DM freeze-in from the decay of long-lived neutral parent particles at the LHC and future colliders. As opposed to electromagnetic/QCD charged LLPs, which leave tracks in the LHC detectors and could be discovered via searches for heavy stable charged particles (see [33] for an analysis of DM freeze-in LHC signatures in these scenarios), neutral LLPs leave no trace in the ATLAS and CMS detectors until their decay. They constitute an ideal search objective for the proposed MATHUSLA surface detector [28, 35-37] and other recent proposals for LLP detectors [38-41].

As a concrete model, we study a freeze-in DM scenario via the SM Higgs (a simple version of a supersymmetric Higgsino-Axino [29, 42, 43] or Higgsino-singlino system, very similar to the so-called singlet-doublet DM scenario from $[32,44])$. We perform an analysis of the projected sensitivity of MATHUSLA in its $100 \mathrm{~m} \times 100 \mathrm{~m}$ (MATHUSLA100) and $200 \mathrm{~m} \times 200 \mathrm{~m}$ (MATHUSLA200) design proposals [37], to the freeze-in DM parameter space, and study its complementarity to LLP searches with the ATLAS and CMS detectors which use displaced vertices and missing transverse energy $E_{T}^{\text {miss }}[32]$. We then analyze the improvement in sensitivity than would come from a forward detector within a future $100 \mathrm{TeV} p p$ - collider $(F C C-h h)$.

In addition, we discuss in detail several technical aspects of the present DM freeze-in scenario (the latter three being in fact more general): the role of the breaking of electroweak (EW) symmetry in the early Universe (the EW phase transition) on the freeze-in production of DM; the inclusion of thermal masses on the computation of the DM relic density, previously disregarded in DM freeze-in studies (there have been however very recent

\footnotetext{
${ }^{1}$ The inverse processes are absent due to the small DM abundance w.r.t. equilibrium densities and to the feeble coupling between DM and the thermal bath.

${ }^{2}$ This is commonly known as infrared freeze-in. For other possibilities, see e.g. [20, 22].

${ }^{3}$ See e.g. [23], although see [17, 24-26] for recent phenomenological probes of freeze-in through a portal.
} 
efforts to include these effects as part of fully-consistent DM freeze-in analyses [17-19]); the potential impact of $2 \rightarrow 2$ scattering processes on setting the DM relic abundance and the interplay between freeze-in and super-WIMP DM production mechanisms (see also [15] for a detailed analysis on the latter). Finally, we analyze the constraints from cosmology, namely from Big-Bang Nucleosynthesis (BBN) and from limits on the washout of small-scale structure via Lyman- $\alpha$ forest observations.

Our work is organized as follows: we give a review of the freeze-in DM production mechanism in section 2. In section 3 we discuss DM freeze-in via the SM Higgs, introduce the model and analyze the DM relic density including the impact of the EW phase transition, of including thermal masses in the calculation and of the super-WIMP DM production mechanism. In section 4 we discuss the constraints from BBN and from Lyman- $\alpha$ forest observations. In section 5 we obtain the projected sensitivity of MATHUSLA to the DM freeze-in parameter space, and in section 6 we analyze its complementarity with ongoing LHC searches: mono-jet, disappearing tracks and displaced vertices $+E_{T}^{\text {miss }}$. In section 7 we derive the future prospects of an FCC-hh $100 \mathrm{TeV}$ collider with a forward detector, and finally we conclude in section 8. Various technical details are confined to the appendices: appendix A discusses $1 \rightarrow 2$ vs $2 \rightarrow 2$ processes regarding the DM relic density. Appendix B presents details of the recasting procedure for the ATLAS displaced vertices $+E_{T}^{\text {miss }}$ search [45]. Appendix C explicitly presents the NLO+NLL Higgsino pair production cross sections obtained with Resummino-2.0.1 [46, 47] and used for our sensitivity estimates throughout the paper.

\section{Dark Matter through the freeze-in mechanism: review}

We review here the main aspects of freeze-in production of DM through the decay of a parent particle $A$ in thermal equilibrium with the plasma [8]

$$
A \rightarrow B_{\mathrm{SM}} \chi
$$

where $B_{\mathrm{SM}}$ is a $\mathrm{SM}$ state and $\chi$ is the $\mathrm{DM}$ candidate. Initially (after reheating at the end of inflation) the DM abundance is assumed to be negligibly small and subsequently increases continuously as the parent particle $A$ within the thermal bath decays into DM during the radiation-dominated era. ${ }^{4}$ This DM production process is effective as long as the parent particle is relativistic. As the temperature of the radiation bath drops below the mass of $A$, the abundance of the parent particle becomes exponentially suppressed and the DM production process ceases to be effective. DM is then said to freeze-in, with most of the $\chi$ particles produced at temperatures $T \sim m_{A} / 3$. The DM number density $n_{\chi}$ evolves according to the Boltzmann equation [8]

$$
\frac{d n_{\chi}}{d t}+3 H n_{\chi}=\Gamma_{A} n_{A}^{\mathrm{eq}} \frac{K_{1}\left(m_{A} / T\right)}{K_{2}\left(m_{A} / T\right)},
$$

\footnotetext{
${ }^{4}$ In this work we assume a standard cosmological history; see [29, 48] for the impact of a modified thermal history of the Universe in freeze-in DM production.
} 
where $\Gamma_{A}$ is the decay rate producing DM in eq. (2.1) and $K_{1,2}(x)$ are the first and second modified Bessel functions of the $2^{\text {nd }}$ kind. The parent particle equilibrium number density $n_{A}^{\mathrm{eq}}$ can be well-approximated by

$$
n_{A}^{\mathrm{eq}} \approx \frac{g_{A} \xi}{2 \pi^{2}} m_{A}^{2} T K_{2}\left(m_{A} / T\right),
$$

where $g_{A}$ counts the spin d.o.f. of $A$ and $\xi=2$ if the decaying particle is not self-conjugate (otherwise $\xi=1$ ). At high temperatures $\left(T \gg m_{A}\right), n_{A}^{\mathrm{eq}} \sim T^{3}$ characteristic of a relativistic species, while it features the Maxwell-Boltzmann exponential suppression for $T \ll m_{A}$.

The Boltzmann equation (2.2) is general, with the details of the cosmological history entering through the Hubble parameter $H$ and the time vs temperature relation. Conservation of entropy $s=\frac{2 \pi^{2}}{45} g_{*}^{s} T^{3}$ (with $g_{*}^{s}$ being the number of effective degrees of freedom contributing to the entropy density) in general makes it convenient to track the evolution of the DM population by defining the comoving number density (yield), $Y_{\chi} \equiv n_{\chi} / s$, which is dimensionless, and on the other hand allows us to relate time and temperature as $d t=-d T /(\bar{H} T)$, where (see e.g. [49])

$$
\bar{H} \equiv \frac{H}{1+\frac{1}{3} \frac{d \ln g_{*}^{s}}{d \ln T}} .
$$

For a radiation-dominated universe the Hubble parameter is $H \simeq 1.66 \sqrt{g_{*}} T^{2} / M_{\mathrm{Pl}}$, where $g_{*}$ is the number of effective relativistic degrees of freedom contributing to the energy density, and $M_{\mathrm{Pl}}=1.2 \times 10^{19} \mathrm{GeV}$ is the Planck mass. Describing the evolution in terms of $x \equiv m_{A} / T$ and the yield $Y_{\chi}$, the Boltzmann equation (2.2) reads

$$
\frac{d Y_{\chi}}{d x}=\frac{\Gamma_{A}}{H x} Y_{A}^{\mathrm{eq}}(x) \frac{K_{1}(x)}{K_{2}(x)},
$$

with $Y_{A}^{\mathrm{eq}}=n_{A}^{\mathrm{eq}} / s$. Assuming that at very high temperatures $T_{R} \gg m_{A}$ the abundance of $\chi$ is vanishing, this equation can be integrated to give

$$
Y_{\chi} \approx \frac{45 g_{A} \xi}{(1.66) 8 \pi^{4}} \frac{M_{\mathrm{Pl}} \Gamma_{A}}{m_{A}^{2}} \int_{x_{\min }}^{x_{\max }} \frac{1+\frac{1}{3} \frac{d \ln g_{*}^{s}(x)}{d \ln x}}{g_{*}^{s}(x) \sqrt{g_{*}(x)}} K_{1}(x) x^{3} d x,
$$

with $x_{\min }=m_{A} / T_{R} \ll 1$ and $x_{\max } \rightarrow \infty$. Far from phase transitions where the number of d.o.f. in the plasma could potentially change abruptly, the DM yield is to a good approximation

$$
Y_{\chi} \approx \frac{135 g_{A} \xi M_{\mathrm{Pl}} \Gamma_{A}}{16 \pi^{3} m_{A}^{2}} \frac{1}{1.66 g_{*}^{s}\left(m_{A} / 3\right) \sqrt{g_{*}\left(m_{A} / 3\right)}} .
$$

Requiring then the relic abundance of $\chi$

$$
\Omega_{\chi} h=\frac{m_{\chi} Y_{\chi}}{\rho_{c} / s_{0}}
$$

(with $\rho_{c} / s_{0}=3.6 \times 10^{-9} \mathrm{GeV}$ the critical energy density over the entropy density today) to match the observed DM relic abundance $\Omega_{\mathrm{DM}} h=0.12$, we can obtain an estimate of 
the corresponding decay length $c \tau_{A}$ of the parent particle:

$$
c \tau_{A} \sim 3.6 \mathrm{~m}\left(\frac{g_{A} \xi}{4}\right)\left(\frac{106.75}{g_{*}}\right)^{3 / 2}\left(\frac{m_{\chi}}{100 \mathrm{keV}}\right)\left(\frac{300 \mathrm{GeV}}{m_{A}}\right)^{2},
$$

where we have assumed for simplicity $g_{*}^{s}(x)=g_{*}(x)$ at $T=m_{A} / 3$ (approximately the freeze-in temperature, see [49]). We note the macroscopic lifetime for the parent particle $A$, which according to eq. (2.9) increases linearly with the DM mass $m_{\chi}$ as a result of requiring $\Omega_{\chi} h=0.12$.

\section{Freeze-in (from decay) via the Standard Model Higgs}

In the present work we concentrate on freeze-in DM scenarios where the parent particle $A$ in eq. (2.1) is neutral, which are significantly more challenging to probe experimentally than those with a charged parent particle, ${ }^{5}$ particularly if the decay length of $A$ is large, $c \tau_{A} \gg 1 \mathrm{~m}$. In the following we consider $B_{\mathrm{SM}}$ in eq. (2.1) to be the SM Higgs [29, 32, 43].

\subsection{The model}

A simple model which features the SM Higgs as $B_{\mathrm{SM}}$ consists on adding to the SM a Dirac fermion $\chi$, singlet under the SM gauge group, and an $\mathrm{SU}(2)_{L}$ Dirac fermion doublet $\Psi$ with hypercharge $1 / 2$

$$
\Psi=\left(\begin{array}{l}
\psi^{+} \\
\psi^{0}
\end{array}\right)
$$

such that the Lagrangian reads:

$$
\mathcal{L}=\mathcal{L}_{\mathrm{SM}}+i \bar{\chi} \gamma^{\mu} \partial_{\mu} \chi+i \bar{\Psi} \gamma^{\mu} D_{\mu} \Psi-m_{s} \bar{\chi} \chi-m_{D} \bar{\Psi} \Psi-y_{\chi} \bar{\Psi} H \chi+\text { h.c. }
$$

This model is very similar to the singlet-doublet DM model considered in [32, 44], and could be regarded as a simplified version of the Higgsino-Axino system studied in [10, 29, 42, 43], or an analog of a would-be feebly interacting Higgsino-Bino or Higgsino-Singlino system, bearing in mind that here $\chi$ and $\Psi$ are Dirac fermions.

The neutral particles $\chi$ and $\psi^{0}$ mix after EW symmetry breaking due to the presence of the $y_{\chi}$ coupling, giving rise to mass eigenstates $\chi_{1}$ (mostly singlet-like) and $\chi_{2}$ (mostly doublet-like), their respective masses being $m_{1}$ and $m_{2}$. We consider $m_{2}>m_{1}$ and the coupling $y_{\chi} \ll 1$, as needed for the freeze-in mechanism to yield the correct DM relic density. In this limit we have $m_{1} \simeq m_{s}$ and $m_{2} \simeq m_{D}$ in eq. (3.2) $\left(m_{2}>m_{1}\right.$ is then achieved by setting $m_{D}>m_{s}$ ). The singlet-doublet mixing is simply given by

$$
\sin \theta \simeq \frac{y_{\chi} v}{\sqrt{2}\left(m_{2}-m_{1}\right)}
$$

valid in the limit $m_{2}-m_{1} \gg y_{\chi} v$, with $v=246 \mathrm{GeV}$ the SM Higgs vev. After EW symmetry breaking, the interactions of the DM candidate $\chi_{1}$ are then given by $h-\chi_{2}-\chi_{1}$,

\footnotetext{
${ }^{5}$ For a detailed phenomenological study of freeze-in scenarios where the parent particle is electrically and/or QCD charged, see [33].
} 

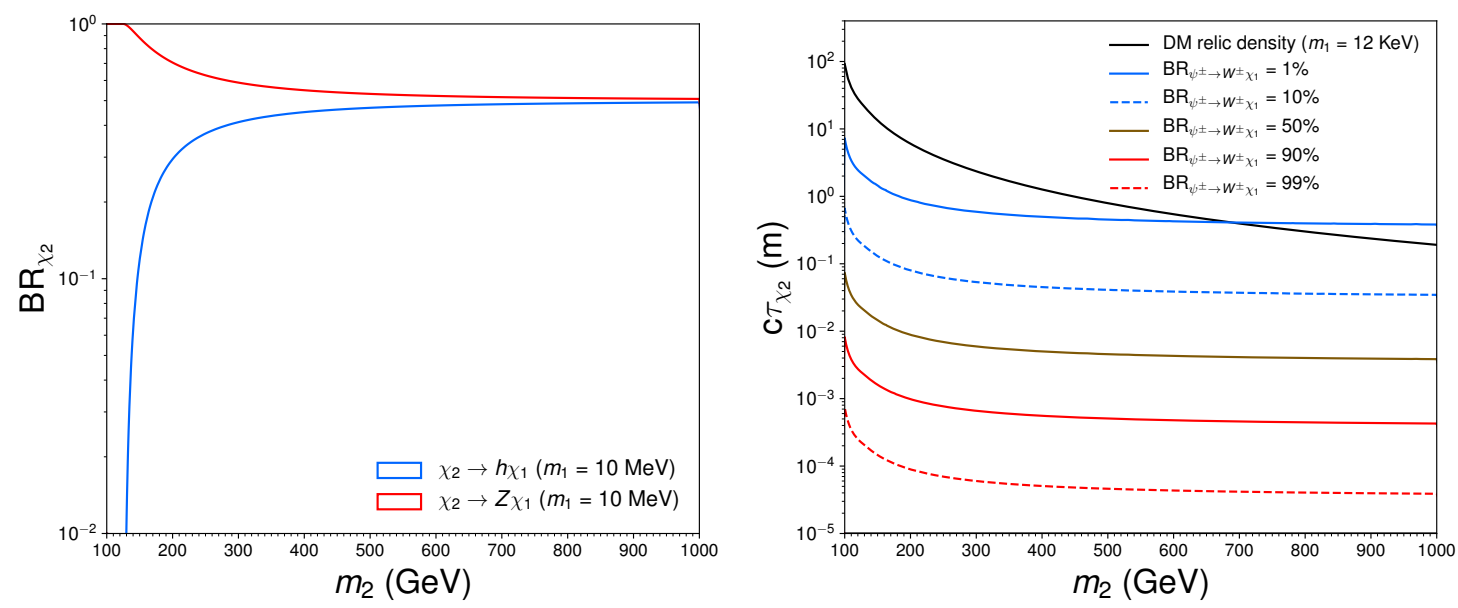

Figure 1. Left: branching fractions for $\chi_{2} \rightarrow h \chi_{1}$ (blue) and $\chi_{2} \rightarrow Z \chi_{1}$ (red) as a function of $m_{2}$ for $m_{1}=10 \mathrm{MeV}$. Right: branching ratio $\mathrm{BR}\left(\psi^{ \pm} \rightarrow W^{ \pm} \chi_{1}\right)$ values in the $\left(m_{2}, c \tau_{\chi_{2}}\right.$ plane for a DM mass $m_{1}=12 \mathrm{KeV}$. The solid black line corresponds to $\Omega_{\chi} h=0.12$ for $m_{1}=12 \mathrm{KeV}$ as computed with micrOMEGAs5.0 [49] (assuming an EW phase transition temperature $T_{\mathrm{EW}}=160 \mathrm{GeV}$, see the discussion in section 3.2 ).

directly from the Yukawa term in eq. (3.2), and $Z-\chi_{2}-\chi_{1}, W^{ \pm}-\psi^{\mp}-\chi_{1}$ from the singlet-doublet mixing induced by the Yukawa term after EW symmetry breaking. When kinematically possible, the decay widths for $\chi_{2} \rightarrow h \chi_{1}, \chi_{2} \rightarrow Z \chi_{1}$ and $\psi^{ \pm} \rightarrow W^{ \pm} \chi_{1}$ are given by

$$
\begin{aligned}
\Gamma\left(\chi_{2} \rightarrow h \chi_{1}\right) & =\frac{y_{\chi}^{2}}{32 \pi m_{2}^{3}}\left[\left(m_{2}+m_{1}\right)^{2}-m_{h}^{2}\right] \lambda\left(m_{2}, m_{1}, m_{h}\right) \\
\Gamma\left(\chi_{2} \rightarrow Z \chi_{1}\right) & =\frac{y_{\chi}^{2}}{32 \pi} \frac{\left[\left(m_{2}-m_{1}\right)^{2}-m_{Z}^{2}\right]\left[\left(m_{2}+m_{1}\right)^{2}+2 m_{Z}^{2}\right]}{m_{2}^{3}\left(m_{2}-m_{1}\right)^{2}} \lambda\left(m_{2}, m_{1}, m_{Z}\right) \\
\Gamma\left(\psi^{ \pm} \rightarrow W^{ \pm} \chi_{1}\right) & =\frac{y_{\chi}^{2}}{32 \pi} \frac{\left[\left(m_{\psi}-m_{1}\right)^{2}-m_{W}^{2}\right]\left[\left(m_{\psi}+m_{1}\right)^{2}+2 m_{W}^{2}\right]}{m_{\psi}^{3}\left(m_{\psi}-m_{1}\right)^{2}} \lambda\left(m_{\psi}, m_{1}, m_{W}\right)
\end{aligned}
$$

with $\lambda(x, y, z)=\sqrt{x^{4}+y^{4}+z^{4}-2 x^{2} y^{2}-2 x^{2} z^{2}-2 y^{2} z^{2}}$. In figure 1 (left) we show the branching fractions of $\chi_{2}$ as a function of $m_{2}$ for a benchmark ${ }^{6} m_{1}=10 \mathrm{MeV}$, highlighting that for $m_{2} \lesssim 400 \mathrm{GeV}$ the decay $\chi_{2} \rightarrow Z \chi_{1}$ starts to dominate over $\chi_{2} \rightarrow h \chi_{1}$. For $m_{2}-m_{1} \rightarrow m_{h}$ the branching fraction into $h \chi_{1}$ becomes negligible, while for $m_{2}-m_{1} \gg m_{h}$ both branching fractions approach $50 \%$.

In addition, loops of $\mathrm{EW}$ gauge bosons induce a radiative mass splitting between the charged state $\psi^{ \pm}$and the (mostly doublet-like) neutral state $\chi_{2}[50,51]$. The value of this mass splitting $\delta m$ is given by

$$
\delta m=m_{\psi^{ \pm}}-m_{2}=\frac{g^{2} m_{Z}}{32 \pi^{2}} \sin ^{2} \theta_{W} f\left(\frac{m_{z}}{m_{2}}\right),
$$

with $g$ the $\mathrm{SU}(2)_{L}$ gauge coupling, $\theta_{W}$ the Weinberg angle and $f\left(m_{Z} / m_{2}\right)$ given by

$$
f(r)=2 r^{3} \log r-2 r+\left(r^{2}+2\right) \sqrt{r^{2}-4} \log \left(\frac{r^{2}-2-r \sqrt{r^{2}-4}}{2}\right) .
$$

\footnotetext{
${ }^{6}$ Compared to the results in [32], ours correspond to the choice $\tan \theta=1$ in [32].
} 
For $m_{2} \in[100,1000] \mathrm{GeV}$ the range of mass splitting values is $\delta m \in[260,340] \mathrm{MeV}$. For such splittings the decay mode $\psi^{ \pm} \rightarrow \pi^{ \pm} \chi_{2}$ completely dominates over the decay involving charged leptons $\psi^{ \pm} \rightarrow \ell^{ \pm} \nu \chi_{2}$ [50], with the width $\Gamma\left(\psi^{ \pm} \rightarrow \pi^{ \pm} \chi_{2}\right)$ given by

$$
\Gamma\left(\psi^{ \pm} \rightarrow \pi^{ \pm} \chi_{2}\right)=\frac{\cos ^{2} \theta_{c}}{2 \pi v^{4}} f_{\pi}^{2} \delta m^{3} \sqrt{1-\frac{m_{\pi}^{2}}{\delta m^{2}}},
$$

with $f_{\pi} \simeq 130 \mathrm{MeV}$ and $\theta_{c}$ the Cabibbo angle. For the very small values of the singletdoublet mixing relevant for DM freeze-in, the decay width from eq. (3.7) is generally much larger than $\Gamma\left(\psi^{ \pm} \rightarrow W^{ \pm} \chi_{1}\right)$ and yields the dominant decay mode for $\psi^{ \pm}$. This can be seen explicitly in figure 1 (right), where we show the branching ratio $\operatorname{BR}\left(\psi^{ \pm} \rightarrow W^{ \pm} \chi_{1}\right)$ as a function of $m_{2} \simeq m_{\psi^{ \pm}}$and the $\chi_{2}$ decay length $c \tau_{\chi_{2}}$ (which can be directly related to $\Gamma\left(\psi^{ \pm} \rightarrow W^{ \pm} \chi_{1}\right)$ by means of eq. (3.4)) for a benchmark DM mass $m_{1}=12 \mathrm{KeV}$. We also show in figure 1 (right) the DM relic density curve for $m_{1}=12 \mathrm{KeV}$ as computed with micrOMEGAs5.0 [49] (see section 3.2 for details); since roughly all parameter space below this curve is excluded by the Lyman- $\alpha$ bound from Cosmology (see section 4 ), figure 1 (right) highlights that, in order to obtain the observed DM relic abundance, $\operatorname{BR}\left(\psi^{ \pm} \rightarrow\right.$ $\left.W^{ \pm} \chi_{1}\right) \ll 0.1$ for $m_{2}<1 \mathrm{TeV}$ is needed in the present freeze-in scenario.

\subsection{DM relic density: the role of EW symmetry breaking \& thermal masses}

As discussed in section 2, the DM relic abundance is obtained via slow $\chi_{1}$ production from the decays of the parent particles $\chi_{2}, \psi^{ \pm}$present in the thermal bath during the radiation-dominated era. For the computation of the DM relic density, we have used micrOMEGAs5.0 [49] with the additional implementation of several important features, which we detail in the following.

Prior to EW symmetry breaking in the early Universe (the EW phase transition), the interactions of DM with the EW gauge bosons are absent, ${ }^{7}$ and the states $\psi^{0}, \psi^{+}$in (3.1) decay via the Yukawa interaction $y_{\chi}$ into the SM Higgs doublet field components and the $\mathrm{DM}$ candidate, $\Psi \rightarrow H \chi$. The correct evaluation of DM production in the EW symmetric phase requires the inclusion of the temperature-dependent (thermal) mass $\Pi(T)$ of the Higgs doublet, given by [52, 53] (see also [54])

$$
\Pi_{H}^{2}(T)=\left[\frac{3 g^{2}}{16}+\frac{g^{\prime 2}}{16}+\frac{y_{t}^{2}}{4}+\frac{\lambda}{2}\right] T^{2} \simeq(0.631 T)^{2},
$$

with $g, g^{\prime}$ the gauge couplings for $\mathrm{SU}(2)_{L}$ and $\mathrm{U}(1)_{Y}, y_{t}$ the top quark Yukawa coupling and $\lambda$ the Higgs quartic coupling. On the other hand, since the parent particle is in equilibrium with the SM, it will also acquire a thermal mass (see e.g. $[55,56])^{8} \Pi_{\Psi}(T)$ :

$$
\Pi_{\Psi}^{2}(T)=\left[\frac{3 g^{2}}{16}+\frac{g^{\prime 2}}{16}\right] T^{2}
$$

\footnotetext{
${ }^{7}$ We are indebted to Thomas Konstandin for discussions on this point.

${ }^{8}$ Here we use the thermal mass of a Higgsino (or analogously, that of a lepton $\mathrm{SU}(2)_{L}$ doublet with hypercharge $Y=1 / 2$ ). We note that the DM coupling of $\Psi$ may be safely neglected for this purpose.
} 
such that before the EW phase transition we consider the mass of $\Psi$ to be

$$
m_{\Psi}(T)=m_{D}+\Pi_{\Psi}(T) \approx m_{D}+0.293 T .
$$

The temperature dependence from both $\Pi_{H}(T)$ and $\Pi_{\Psi}(T)$ will then be inherited by the parent decay width in the EW symmetric phase $^{9}$

$$
\Gamma_{A}\left(T>T_{\mathrm{EW}}\right)=\Gamma_{\Psi \rightarrow H \chi}(T),
$$

with $T_{\mathrm{EW}}$ the EW phase transition temperature, corresponding to $T_{\mathrm{EW}} \approx 160 \mathrm{GeV}$ in the $\mathrm{SM}[57,58]$. Since the thermal mass of the particle $\Psi$ is smaller than the thermal mass of the Higgs, we have that for $T \gg m_{D}\left(\simeq m_{2}\right)$ the decay $\Psi \rightarrow H \chi$ will not be kinematically open. When this is the case, it is instead the Higgs decay $H \rightarrow \Psi \chi$ which becomes kinematically open and sources DM production, its rate being $\Gamma(H \rightarrow \Psi \chi) \simeq \Gamma(\Psi \rightarrow H \chi) \times\left(m_{\Psi} / \Pi_{H}\right)^{3}$ (with $m_{\Psi}$ and $\Pi_{H}$ given respectively by (3.10) and (3.8)). The temperature at which this change in DM production process approximately occurs is $T \simeq 2.96 m_{2}$. For lower temperatures (yet generally above $T_{\mathrm{EW}}$ ), the decay $\Psi \rightarrow H \chi$ sources DM production and yields the (by far) leading contribution to the DM relic density prior to the EW phase transition.

After the EW phase transition the decays $\chi_{2} \rightarrow Z \chi_{1}$ and $\psi^{ \pm} \rightarrow W^{ \pm} \chi_{1}$ become possible and source DM production together with the decay $\chi_{2} \rightarrow h \chi_{1}$. The decay width $\Gamma_{A}$ of the parent particle(s) responsible for DM freeze-in is in this case given by

$$
\Gamma_{A}\left(T<T_{\mathrm{EW}}\right)=\Gamma\left(\chi_{2} \rightarrow h \chi_{1}\right)+\Gamma\left(\chi_{2} \rightarrow Z \chi_{1}\right)+\Gamma\left(\psi^{ \pm} \rightarrow W^{ \pm} \chi_{1}\right),
$$

where for simplicity we do not consider the temperature dependence of the Higgs vev after EW symmetry breaking and also neglect thermal corrections for temperatures below the EW phase transition.

The temperature dependence of the freeze-in decay processes from eqs. (3.11) and (3.12) has been implemented in a modified version of micrOMEGAs5.0, and in the remainder of this work we consider the range between $T_{\mathrm{EW}}=160 \mathrm{GeV}$ and $T_{\mathrm{EW}}=50 \mathrm{GeV}$ (the latter corresponding to a generic benchmark for a strongly super-cooled first order EW phase transition) when discussing/computing the freeze-in DM relic density.

Both the EW phase transition and the inclusion of thermal masses in the calculation can then have a notable influence on the DM freeze-in process. ${ }^{10}$ In particular, the DM production prior to the EW phase transition can be strongly affected by thermal corrections. The production of DM after the EW phase transition is however not expected to be significantly affected, and as discussed above we are for simplicity neglecting here the thermal effects below $T_{\mathrm{EW}}$. It becomes clear that thermal effects will be more important

\footnotetext{
${ }^{9}$ With the corresponding replacements $m_{h}=\Pi_{H}(T)$ and $m_{2} \rightarrow m_{2}+\Pi_{\Psi}(T)$. Note that $\Gamma_{\Psi \rightarrow H \chi}(T)$ approximately corresponds to four times the value of $\Gamma\left(\chi_{2} \rightarrow h \chi_{1}\right)$ due to the number of degrees of freedom inside the $\Psi$ and SM Higgs doublets.

${ }^{10}$ The potential influence of the EW phase transition on freeze-in processes has already been noted and discussed, albeit for different freeze-in scenarios to the freeze-in from decay $A \rightarrow B_{\mathrm{SM}} \chi$ considered in this work, in refs. [16-18, 59].
} 

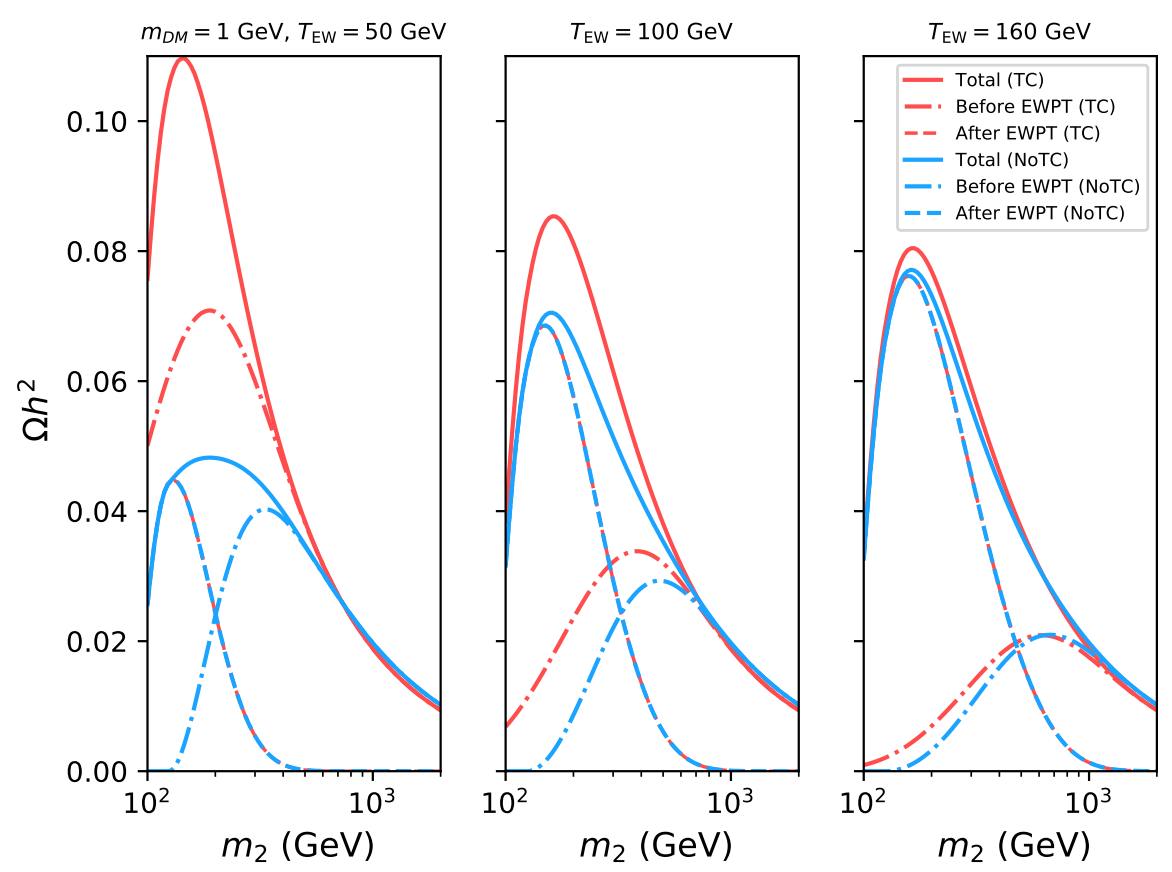

Figure 2. DM Relic density $\Omega h^{2}$ as a function of $m_{2}$, for $m_{1}=1 \mathrm{GeV}, y_{\chi}=10^{-11}$ and $T_{\mathrm{EW}}=$ $50 \mathrm{GeV}$ (left panel), $T_{\mathrm{EW}}=100 \mathrm{GeV}$ (middle panel), $T_{\mathrm{EW}}=160 \mathrm{GeV}$ (right panel). Red lines show the value of $\Omega h^{2}$ from DM production including thermal corrections: before the EW Phase Transition (EWPT, dot-dashed line), after the EWPT (dashed line), and the total amount (solid line). Light-blue lines show the corresponding value of $\Omega h^{2}$ disregarding thermal corrections: from DM production before the EWPT (dot-dashed), after the EWPT (dashed), and total (solid).

when the contribution to the total DM relic density from DM production for $T>T_{\mathrm{EW}}$ is sizable. In figure 2 we show the DM relic density with the inclusion of thermal corrections $\Omega h_{\mathrm{TC}}^{2}$ and the corresponding DM relic density when these are disregarded ${ }^{11} \Omega h_{\mathrm{NoTC}}^{2}$, as well as the partial contributions before and after the EW phase transition, as a function of $m_{2}$ for $m_{1}=1 \mathrm{GeV}$ and $T_{\mathrm{EW}}=50 \mathrm{GeV}$ (left panel), $T_{\mathrm{EW}}=100 \mathrm{GeV}$ (middle panel) and $T_{\mathrm{EW}}=160 \mathrm{GeV}$ (right panel).

As seen in figure 2 , for $m_{2} \gg m_{h}+m_{1}, T_{\mathrm{EW}}$ the inclusion of thermal masses yields a very mild suppression $\Omega h_{\mathrm{TC}}^{2} / \Omega h_{\mathrm{NoTC}}^{2} \sim 0.9$. In this case, DM freezes-in while the EW symmetry is restored, and the broken phase decays $\chi_{2} \rightarrow Z \chi_{1}$ and $\psi^{ \pm} \rightarrow W^{ \pm} \chi_{1}$ do not contribute to DM production in the early Universe. In this regime we find the DM relic abundance to be rather insensitive to the value of $T_{\mathrm{EW}}$. On the other hand, figure 2 shows that the DM production in the EW broken phase starts becoming appreciable for $m_{2} \lesssim 6 T_{\mathrm{EW}}$, and for yet smaller $m_{2}$ the DM production in the broken phase quickly becomes dominant when $T_{\mathrm{EW}} \gtrsim m_{h}$. In contrast, for $T_{\mathrm{EW}} \lesssim m_{h}$ the DM production prior to the EW phase transition is still very important for smaller $m_{2}$ masses, and it is in this region where the inclusion of the thermal corrections yields the largest effect, providing a significant enhancement to the DM relic density compared to the value neglecting thermal

\footnotetext{
${ }^{11}$ Here thermal corrections are disregarded by setting $\Gamma_{\Psi \rightarrow H \chi} \simeq 4 \times\left.\Gamma\left(\chi_{2} \rightarrow h \chi_{1}\right)\right|_{m_{h}=125 \mathrm{GeV}}$.
} 


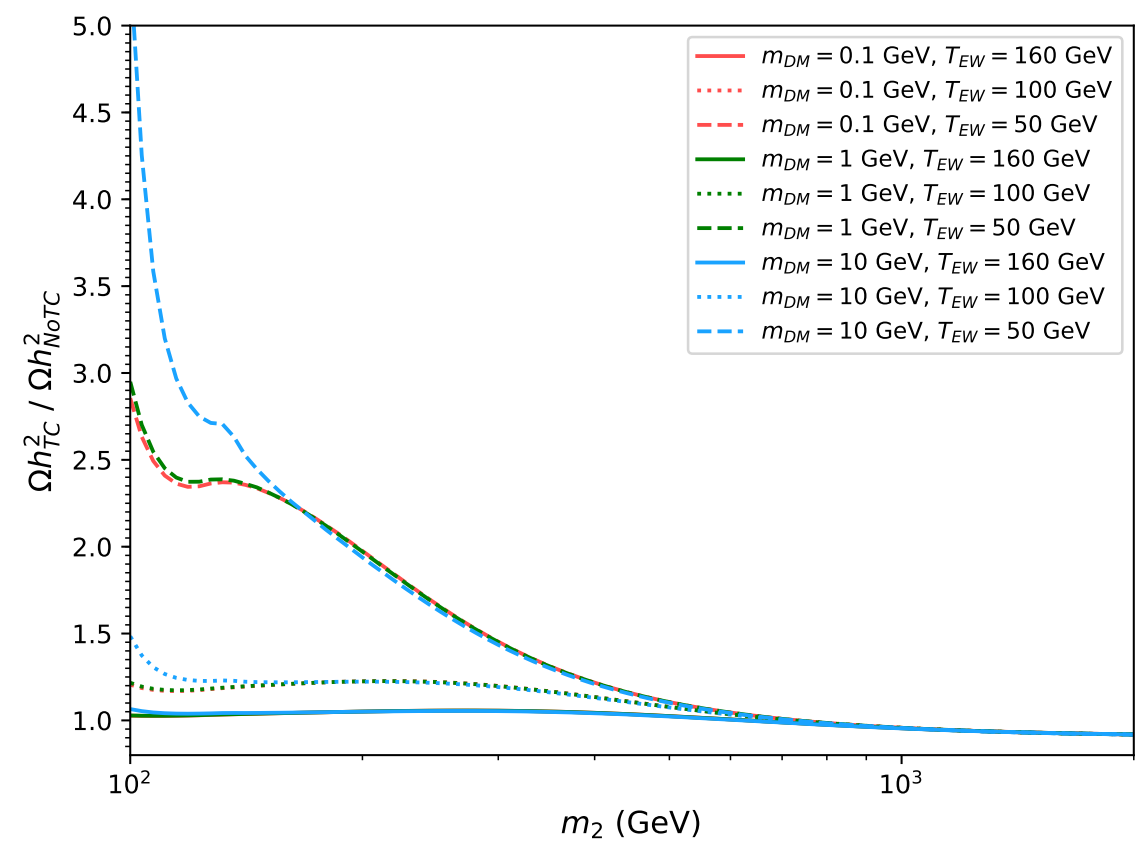

Figure 3. Ratio $\Omega h_{\mathrm{TC}}^{2} / \Omega h_{\mathrm{NoTC}}^{2}$ as a function of $m_{2}$ for a DM mass $m_{1}=0.1 \mathrm{GeV}$ (red), $m_{1}=1 \mathrm{GeV}$ (green) and $m_{1}=10 \mathrm{GeV}$ (light-blue), respectively for $T_{\mathrm{EW}}=160 \mathrm{GeV}$ (solid line), $T_{\mathrm{EW}}=100 \mathrm{GeV}$ (dotted line) and $T_{\mathrm{EW}}=50 \mathrm{GeV}$ (dashed line).

corrections. The difference in behaviour of the two regimes $\left(T_{\mathrm{EW}}\right.$ much above or below the Higgs mass) is due to the fact that the latter case allows for the decay process prior to the EW phase transition to be kinematically open for a longer period. All this may be seen explicitly in figure 3 where $\Omega h_{\mathrm{TC}}^{2} / \Omega h_{\mathrm{NoTC}}^{2}$ is shown as a function of $m_{2}$ for several values of $m_{1}$ and $T_{\mathrm{EW}}$. Note that below $m_{2} \lesssim m_{h}$ the enhancement to the DM relic abundance coming from thermal corrections is significantly larger for $m_{\mathrm{DM}}=10 \mathrm{GeV}$ than for $m_{\mathrm{DM}}=1 \mathrm{GeV}$ or less. This is due to phase space arguments, the parent decay being open or closed in the EW symmetric phase depending on the inclusion of thermal corrections or not, mostly for the lowest $T_{\mathrm{EW}}$ considered.

Finally, we stress that in the above discussion, and throughout the general discussion of freeze-in from decay in [8], outlined in section 2, it has been implicit that $(1 \rightarrow 2)$ decays are the dominant processes producing DM. We nevertheless note that there are contributions to DM production at the same order in the feeble coupling $y_{\chi}$ from $2 \rightarrow 2$ scattering processes such as $q \chi_{2} \rightarrow q \chi_{1}$ (with $q$ a SM quark) mediated by a Higgs boson in the $t$-channel. In a similar manner, for $T<T_{\mathrm{EW}}$ analogous $q \chi_{2} \rightarrow q \chi_{1}$ and $q \chi^{ \pm} \rightarrow q^{\prime} \chi_{1}$ scattering processes with a $Z$ and $W$ boson in the $t$-channel become possible. These processes are discussed in detail ${ }^{12}$ in appendix $\mathrm{A}$, where we find that decays $A \rightarrow B_{\mathrm{SM}} \chi$

\footnotetext{
${ }^{12}$ Appendix A does however not analyze in detail the impact of other $2 \rightarrow 2$ processes in the present freeze-in scenario involving SM gauge bosons $V_{\mathrm{SM}}$, such as $H \Psi \rightarrow \chi V_{\mathrm{SM}}$ and $V_{\mathrm{SM}} \Psi \rightarrow \chi H$. While such processes may be quantitatively more important than scattering processes $q \Psi \rightarrow q \chi$ [60], they are still expected to be highly subdominant to $1 \rightarrow 2$ decays. A precise assessment of the impact of these processes is left for future work.
} 
completely dominate over scatterings $X_{\mathrm{SM}} A \rightarrow Y_{\mathrm{SM}} \chi$ (with $X_{\mathrm{SM}}$ and $Y_{\mathrm{SM}}$ SM particles, and $B_{\mathrm{SM}}$ in the $t$-channel of the scattering process) except when the former are strongly phase-space suppressed, i.e. $m_{B_{\mathrm{SM}}}+m_{\chi} \rightarrow m_{A}$. In addition, other contributions like soft gauge interaction processes (in $1 \rightarrow 2$ and $2 \rightarrow 2$ DM production processes) before the $\mathrm{EW}$ phase transition (see [60] for a discussion) are not expected to be significant in the present case (contrary to e.g. the Type-I see-saw scenario [60]), due to the specific mass spectrum of our freeze-in scenario, with $m_{2}>100 \mathrm{GeV}\left(\gg m_{\ell}\right)$ and $m_{2} \gg m_{\chi} \cdot{ }^{13}$

Finally, scatterings do become dominant in the region $m_{B_{\mathrm{SM}}}+m_{\chi}>m_{A}>m_{\chi}$ where $1 \rightarrow 2$ decays are kinematically forbidden and instead $A$ decays ${ }^{14}$ via a 3 -body process. In our model, the latter situation occurs for $m_{2}-m_{1}<m_{W}$, when all the decays of $\chi_{2}$ and $\psi^{ \pm}$that produce DM are 3-body (e.g. $\chi_{2} \rightarrow b \bar{b} \chi_{1}$ through an off-shell Higgs boson). Since we do not consider these parameter space regions in the present work due to experimental constraints (e.g. the region $m_{\psi^{ \pm}}<m_{W}$ is strongly constrained by LEP [61, 62]), $2 \rightarrow 2$ scattering processes can be safely disregarded in our scenario.

\subsection{Super-WIMP contribution to the DM relic abundance}

For decay widths of the parent particle significantly smaller than its inverse freeze-out timescale

$$
\Gamma_{A} \ll \frac{\left(m_{A} / 20\right)^{2}}{\sqrt{\left(90 /\left(32 \pi^{3} g_{*}\right)\right)} M_{\mathrm{Pl}}} \sim 0.085\left(\frac{m_{A}}{M_{\mathrm{Pl}}}\right) \times m_{A},
$$

which in the present scenario roughly corresponds to $c \tau_{\chi_{2}} \gg\left(100 \mathrm{GeV} / m_{2}\right)^{2} \times 3$ meters, the thermal freeze-out of $\chi_{2}$ followed by its decay into $\chi_{1}$ will also contribute to the DM relic density. The contribution to the DM relic abundance from this mechanism is given by $\Omega_{2} h^{2} \times m_{1} / m_{2}$, where $\Omega_{2} h^{2}$ is the freeze-out abundance of $\chi_{2}$. Assuming that $\chi_{2}$ undergoes freeze-out before decaying as implied by eq. (3.13), the freeze-out abundance of $\chi_{2}$ corresponds to that of thermal Higgsino DM, which for $m_{2} \gg m_{W}$ is simply given by [63]

$$
\Omega_{2} h^{2} \simeq 0.1\left(\frac{m_{2}}{\mathrm{TeV}}\right)^{2}
$$

When $\Omega_{2} h^{2} \times m_{1} / m_{2}$ yields the dominant contribution to the DM relic density, it is usually referred to as the super-WIMP [64] mechanism (see also [15] for a recent work on the interplay between the freeze-in and super-WIMP scenarios). Combining the super-WIMP and freeze-in contributions to the DM relic density, we get the total DM relic abundance

$$
\Omega_{\mathrm{DM}} h^{2}=\Omega_{1} h^{2}+\Omega_{2} h^{2} \times \frac{m_{1}}{m_{2}} .
$$

The above discussion allows to quantify the relative importance of the super-WIMP and freeze-in contributions to the DM relic abundance, with their ratio (SW/FI) given by $\left(m_{1} \Omega_{2} h^{2}\right) /\left(m_{2} \Omega_{1} h^{2}\right)$. In figure 4 we show the SW/FI ratio in the $\left(m_{2}, \mathrm{c} \tau_{\chi_{2}}\right)$ plane, fixing here for simplicity $T_{\mathrm{EW}}=160 \mathrm{GeV}$ and two benchmark values for the DM mass, $m_{1}=$

\footnotetext{
${ }^{13} \mathrm{~A}$ detailed analysis of these processes in freeze-in scenarios of the type discussed in this paper is however necessary to quantitatively assess this statement, and still lacking in the literature.

${ }^{14}$ We assume that $B_{\mathrm{SM}}$ is itself unstable, otherwise for $m_{B_{\mathrm{SM}}}+m_{\chi}>m_{A}$ the state $A$ becomes stable.
} 


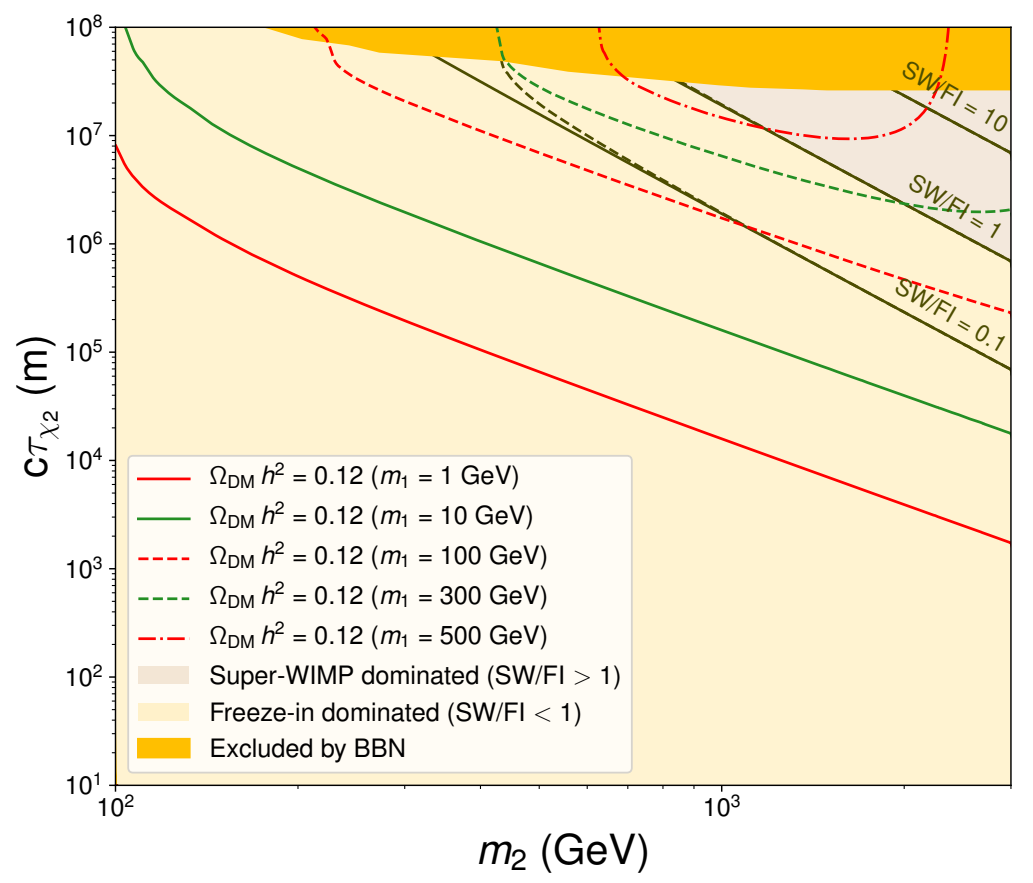

Figure 4. Values of the SW/FI ratio $\left(m_{1} \Omega_{2} h^{2}\right) /\left(m_{2} \Omega_{1} h^{2}\right)$ in the $\left(m_{2}, \mathrm{c} \tau_{\chi_{2}}\right)$ plane for $m_{1}=1 \mathrm{GeV}$ (solid black lines) and $m_{1}=300 \mathrm{GeV}$ (dashed black lines). The red/green lines correspond to the DM relic abundance condition $\Omega_{\mathrm{DM}} h^{2}=0.12$ for various choices of the DM mass $m_{1}$. The Yellow region is excluded by BBN (see section 4 )

$1 \mathrm{GeV}$ and $m_{1}=300 \mathrm{GeV}$ (we nevertheless note that the SW/FI ratio is approximately independent of $m_{1}$, except for $m_{2}-m_{1} \rightarrow m_{h}$, as can be seen in figure 4). We also show in figure 4 the corresponding DM relic abundance condition $\Omega_{\mathrm{DM}} h^{2}=0.12$ using micrOMEGAs5. 0 and eq. (3.15) for various choices of the DM mass $m_{1}$.

As is apparent from eq. (3.14), in the present scenario the super-WIMP mechanism could only account for a significant fraction of the observed DM relic density for $m_{2}>$ 1.1 TeV. In addition large DM masses $m_{1} \gtrsim 300 \mathrm{GeV}$ are required, as shown in figure 4 and expected in order to partially overcome the otherwise large suppression factor $m_{1} / m_{2}$ in the super-WIMP contribution from (3.15). Such large DM masses imply large values of $c \tau_{\chi_{2}}$ for DM not to be overproduced by the freeze-in mechanism, which in turn may become constrained from Big Bang Nucleosynthesis (BBN). We discuss these constraints in the next section.

\section{Constraints on Dark Matter freeze-in from cosmology}

As previously mentioned, there are several important cosmological constraints on this class of freeze-in DM scenarios (for a discussion of such constraints in similar scenarios, see also $[32,33])$. The first constraint we need to consider comes from Big Bang Nucleosynthesis $(\mathrm{BBN})$, which accurately explains the measured primordial abundances of light elements in the Universe ${ }^{15}$ (see e.g. $[66,67]$ ). If the state $\chi_{2}$ lives long enough to decay during BBN,

\footnotetext{
${ }^{15}$ With the only potential exception of ${ }^{7} \mathrm{Li}$, see e.g. [65] for a discussion.
} 
its visible decay products may induce several processes that alter the predictions of $\mathrm{BBN}^{16}$ In order to obtain the corresponding bounds on the parameter space of $m_{1}, m_{2}$ and $\mathrm{c} \tau_{\chi_{2}}$, we use the recent re-analysis of BBN constraints on long-lived decaying particles from [69]. Concerning the hadronic final states $\bar{q} q$ (with $q$ either a first/second generation quark or a $b$ quark), ref. [69] derives bounds on the amount of energy injected in the radiation bath in the form of hadrons from the decay of a long-lived particle $X$, as a function of the particle lifetime $\tau_{X}$ and under the assumption that $X$ decays dominantly into $\bar{q} q,{ }^{17}$ meaning that the hadronic energy injection per particle decay is $E_{\bar{q} q} \sim m_{X}$. In the present case the hadronic energy injection from the decay of $\chi_{2}$ corresponds only to the energy fraction carried by the visible decay products of $\chi_{2}$, either $h$ or $Z$ (both decays $\chi_{2} \rightarrow h \chi_{1}$ and $\chi_{2} \rightarrow Z \chi_{1}$ are present during the BBN epoch, which occurs for temperatures much below the EW phase transition temperature $\left.T_{\mathrm{EW}}\right)$. This is given by

$$
\begin{array}{ll}
E_{\bar{q} q} \simeq \sqrt{m_{h}^{2}+\frac{m_{2}^{2}}{4}\left[\left(1-\xi_{h}-\xi_{1}\right)^{2}-4 \xi_{h} \xi_{1}\right]} & \left(\chi_{2} \rightarrow h \chi_{1}\right) \\
E_{\bar{q} q} \simeq \sqrt{m_{Z}^{2}+\frac{m_{2}^{2}}{4}\left[\left(1-\xi_{z}-\xi_{1}\right)^{2}-4 \xi_{z} \xi_{1}\right]} & \left(\chi_{2} \rightarrow Z \chi_{1}\right)
\end{array}
$$

with $\xi_{h}=m_{h}^{2} / m_{2}^{2}, \xi_{z}=m_{Z}^{2} / m_{2}^{2}$ and $\xi_{1}=m_{1}^{2} / m_{2}^{2}$. From [69] we then obtain the limits on the total hadronic energy injection onto the thermal bath from $\chi_{2}$ decays, given by

$$
\frac{\Omega_{2} h^{2}}{m_{2}} \times \sum_{a=h, Z} E_{\bar{q} q}^{a} \times \operatorname{BR}\left(\chi_{2} \rightarrow a \chi_{1}\right) \operatorname{BR}(a \rightarrow \text { hadrons })
$$

with $\Omega_{2} h^{2}$ from (3.14). Assuming $m_{1} \ll m_{2}, m_{h}$, the corresponding limits on the $\left(m_{2}\right.$, $\mathrm{c} \tau_{\chi_{2}}$ ) parameter space are shown in figure 5 (left). We note that leptonic decays of $\chi_{2}$ also affect BBN, yet these constraints are found to be significantly weaker than the hadronic ones (see [69]) and are therefore not relevant in the present case.

A second important set of limits originate from constraints on the washout of smallscale structure by DM with a non-negligible velocity dispersion (partially relativistic, or warm). The leading such constraint comes from Lyman- $\alpha$ forest observations. For thermal warm dark matter (WDM) these observations place a lower limit on the DM mass in the range $m_{\mathrm{DM}} \gtrsim 4.09-5.3 \mathrm{keV}$ [70-72]. In two recent works [73, 74] (see also [75, 76]) the Lyman- $\alpha$ limit for the case of freeze-in DM produced via two-body decays of a parent particle in thermal equilibrium with the plasma (which is precisely our scenario) has been estimated by comparing the suppression in the linear matter power spectrum from a thermal WDM scenario (with $m_{\mathrm{DM}}$ given by the WDM Lyman- $\alpha$ limit, taken to be $4.65 \mathrm{keV}[71,77])$ to that of the freeze-in scenario. The Lyman- $\alpha$ bound in this case reads

$$
m_{\mathrm{DM}} \gtrsim 12 \mathrm{keV}\left(\frac{\sum_{i j} g_{i} \Gamma_{i j} \Delta_{i j}^{\eta}}{\sum_{i j} g_{i} \Gamma_{i j}}\right)^{1 / \eta},
$$

\footnotetext{
${ }^{16}$ For even longer lifetimes $\tau \gtrsim 10^{6}$ seconds, constraints from the Cosmic Microwave Background (CMB) spectral distorsion become very strong (see [68, 69]). Such long lifetimes are however not relevant for our study.

${ }^{17}$ The corresponding limits for the $\bar{b} b$ and $\bar{u} u$ final states found in [69] are very similar, and thus approximately valid for scenarios where both decays into $b$-quark pairs and light-quark pairs are possible.
} 

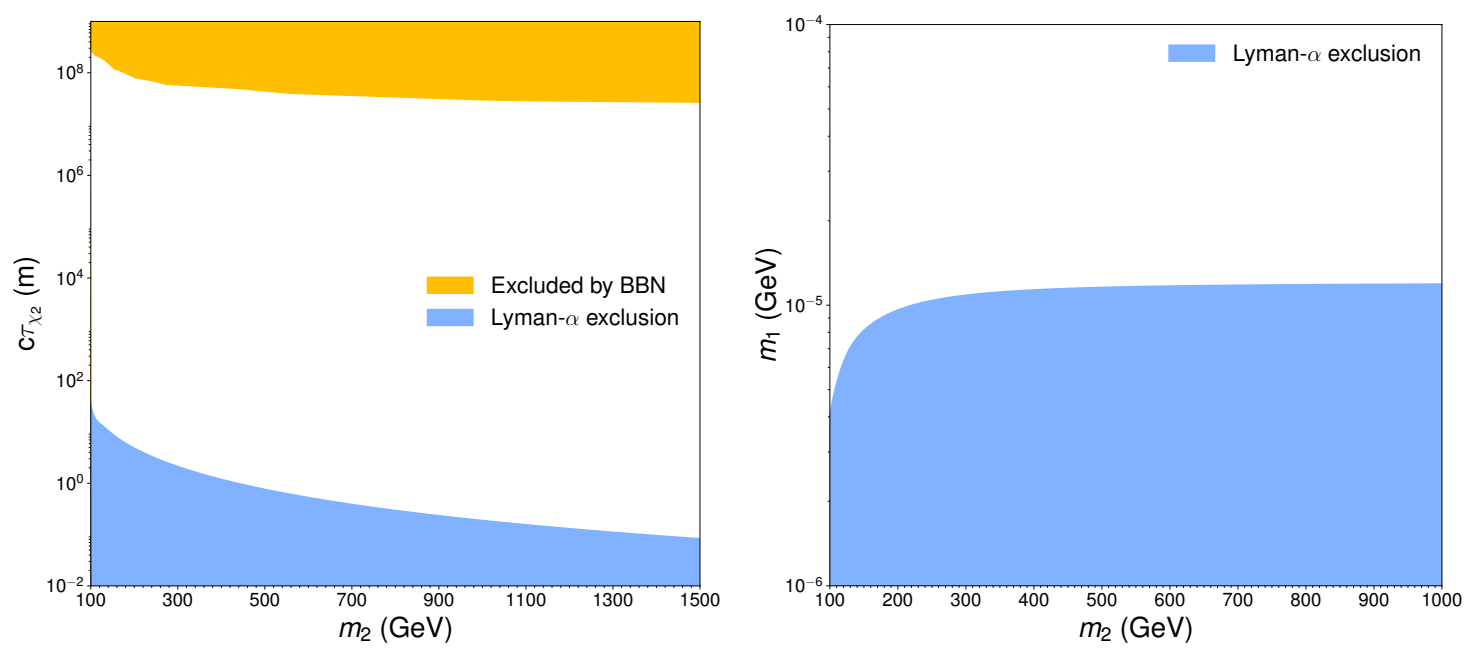

Figure 5. Left: region in the $\left(m_{2}, c \tau_{\chi_{2}}\right)$ plane excluded from Big Bang Nucleosynthesis (yellow) and from Lyman- $\alpha$ forest observations (blue). Right: Lyman- $\alpha$ bound on $m_{1}$ (blue) from eq. (4.3) as a function of $m_{2}$.

where the sum runs over all decay channels of the parent particle(s) that contribute to DM production, $\Gamma_{i j}$ are the corresponding decay widths $\Gamma\left(A_{i} \rightarrow B_{j} \chi_{1}\right)$ (in our case given by eq. (3.4)), $g_{i}$ are the number of degrees of freedom of the parent particle $A_{i}$, the parameter $\Delta_{i j}$ yields the mass splitting between the parent particle and the visible decay product in each channel (e.g. $\Delta_{i j}=1-m_{Z}^{2} / m_{2}^{2}$ for the decay $\chi_{2} \rightarrow Z \chi_{1}$ ) and $\eta \simeq 1.9$ [74]. Assuming that freeze-in DM saturates the observed DM relic density, figure 5 (right) shows the Lyman- $\alpha$ bound on $m_{1}$ in the present scenario. This bound can in turn be translated into a lower limit for $c \tau_{\chi_{2}}$, shown in figure 5 (left).

The combination of BBN and Lyman- $\alpha$ bounds yields an allowed range of parent particle decay lengths $c \tau_{\chi_{2}}$ for viable DM freeze-in, $c \tau_{\chi_{2}} \sim 10^{-1}-10^{7}$ meters, which provide a clear target for LLP searches at colliders. We investigate the current and projected sensitivity of such searches to the Higgs-assisted freeze-in from decay DM scenario analyzed in this work in sections $5-7$.

\section{Probing freeze-in Dark Matter with the MATHUSLA detector}

Considering the limit $m_{2} \gg m_{1}, m_{W}, m_{Z}, m_{h}$ in combination with the freeze-in DM relic abundance condition $\Omega_{\mathrm{DM}} h=0.12$ yields an estimate for the decay length of $\chi_{2}$ (see also eq. (2.9))

$$
c \tau_{\chi_{2}} \sim 4 \mathrm{Km} \times\left(\frac{m_{1}}{100 \mathrm{MeV}}\right)\left(\frac{500 \mathrm{GeV}}{m_{2}}\right)^{2} .
$$

Such long lifetimes make it challenging to search for $\chi_{2}$ with the LHC's ATLAS and CMS detectors (we will nevertheless discuss the current ATLAS and CMS bounds, as well as future prospects in section 6), except maybe for rather small values of $m_{1}$ close to the Lyman- $\alpha$ bound [32]. Here we analyze the prospects for probing the parameter space of the present scenario with the proposed MATHUSLA surface detector for long-lived 
particles [28, 35-37]. The large detector volume of MATHUSLA together with its capability to effectively operate as a background-free environment for LLP searches (see $[28,37]$ for details) at the LHC, yields an ideal setup to probe freeze-in DM scenarios.

At the LHC, the states $\chi_{2}$ and $\psi^{ \pm}$can be produced via the Drell-Yan processes $p p \rightarrow$ $\chi_{2} \chi_{2}, p p \rightarrow \chi_{2} \psi^{ \pm}, p p \rightarrow \psi^{+} \psi^{-}$. For our study, we implement the Lagrangian from eq. (3.2) in FeynRules [78] and simulate the various Drell-Yan production processes for $\chi_{2}$ and $\psi^{ \pm}$ at $\sqrt{s}=13 \mathrm{TeV}$ LHC in Madgraph_aMC@NLO [79]. We then normalize the respective cross sections to the corresponding $\sqrt{s}=13 \mathrm{TeV}$ LHC NLO+NLL charged/neutral Higgsino production cross sections $\sigma_{13}^{\mathrm{LHC}}$ computed with Resummino-2.0.1 $[46,47]^{18}$ (with the PDF set MSTW2008NLO90CL from LHAPDF [81]). The Higgsino pair production cross sections for the various Drell-Yan processes are shown explicitly in appendix C. In the following, we also consider that the decays $\psi^{ \pm} \rightarrow \chi_{2}+X\left(X=\pi^{ \pm}, \ell^{ \pm} \nu\right.$ being very soft $)$, which occur within a few $\mathrm{cm}$ of the interaction point, effectively convert all the charged states $\psi^{ \pm}$into neutral $\chi_{2}$ ones with approximately identical kinematics.

The MATHUSLA detector would be located at a distance $\sim \mathcal{O}(100 \mathrm{~m})$ from the ATLAS or CMS interaction point. The probability for a $\chi_{2}$ particle to decay inside the MATHUSLA detector volume is given by

$$
P_{\text {decay }}^{\mathrm{MATH}}=\epsilon_{\text {geometric }} \times P_{\text {decay }}\left(\beta c \tau_{\chi_{2}}, L_{a}, L_{b}\right)
$$

with $P_{\text {decay }}\left(\beta c \tau_{\chi_{2}}, L_{a}, L_{b}\right)$ given by

$$
P_{\text {decay }}\left(\beta c \tau_{\chi_{2}}, L_{a}, L_{b}\right)=e^{-\frac{L_{a}}{\beta c \tau} \chi_{2}}-e^{-\frac{L_{b}}{\beta c \tau} \chi_{2}} .
$$

Here $L_{a}$ and $L_{b}$ are the distances from the interaction point for which $\chi_{2}$ respectively enters and leaves the MATHUSLA detector volume, and $\beta$ is the $\chi_{2}$ boost factor $\left|\vec{p}_{\chi_{2}}\right| / m_{2}$. In this work we consider two possible MATHUSLA design configurations: MATHUSLA100 and MATHUSLA200. In the former case, the MATHUSLA detector volume for LLP decays as measured from the interaction point is [37] $x \in[-50,50] \mathrm{m}, y \in[100,120] \mathrm{m}, z \in[100,200]$ $\mathrm{m}$, while in the latter case (corresponding to the original MATHUSLA proposal $[35,37]$ ) the detector volume as measured from the interaction point is $x \in[-100,100] \mathrm{m}, y \in[100,120]$ $\mathrm{m}, z \in[100,300] \mathrm{m}$. The MATHUSLA geometric acceptance $\epsilon_{\text {geometric }}$ in eq. (5.2) then measures the fraction of generated events contained within the MATHUSLA solid angle as seen from the interaction point, which we extract from our event simulation. ${ }^{19}$ Specifically, for the computation of $\epsilon_{\text {geometric }}$ we consider events for which the $\chi_{2}$ trajectory intersects the MATHUSLA tracking layers (see $[35,36]$ for details on the tracking layout within the MATHUSLA detector) and assume for simplicity that the visible $\chi_{2}$ decay products would then also hit the tracking layers if the decay happens inside MATHUSLA (for $m_{2} \gg$ $m_{h}+m_{1}$ this occurs automatically, since the decay products of $\chi_{2}$ will be fairly collinear

\footnotetext{
${ }^{18}$ These are given also by the CERN LHC SUSY XS Working Group [80].

${ }^{19}$ For an isotropic distribution of $\chi_{2} \bar{\chi}_{2}$ events, $\epsilon_{\text {geometric }}$ would correspond to twice the MATHUSLA detector solid angle from the interaction point, $\epsilon_{\text {geometric }} \simeq 2 \times \frac{1}{4 \pi} \int_{-\pi / 4}^{\pi / 4} d \phi \int_{\arctan (12 / 30)}^{\pi / 4} \sin \theta d \theta \sim 0.05$ for MATHUSLA200 and $\epsilon_{\text {geometric }} \simeq 2 \times \frac{1}{4 \pi} \int_{-\arctan (1 / 2)}^{\arctan (1 / 2)} d \phi \int_{\arctan (12 / 20)}^{\pi / 4} \sin \theta d \theta \sim 0.022$ for MATHUSLA100. In a more general case the convolution with the $\eta, \phi$ dependence of the event sample modifies this estimate.
} 


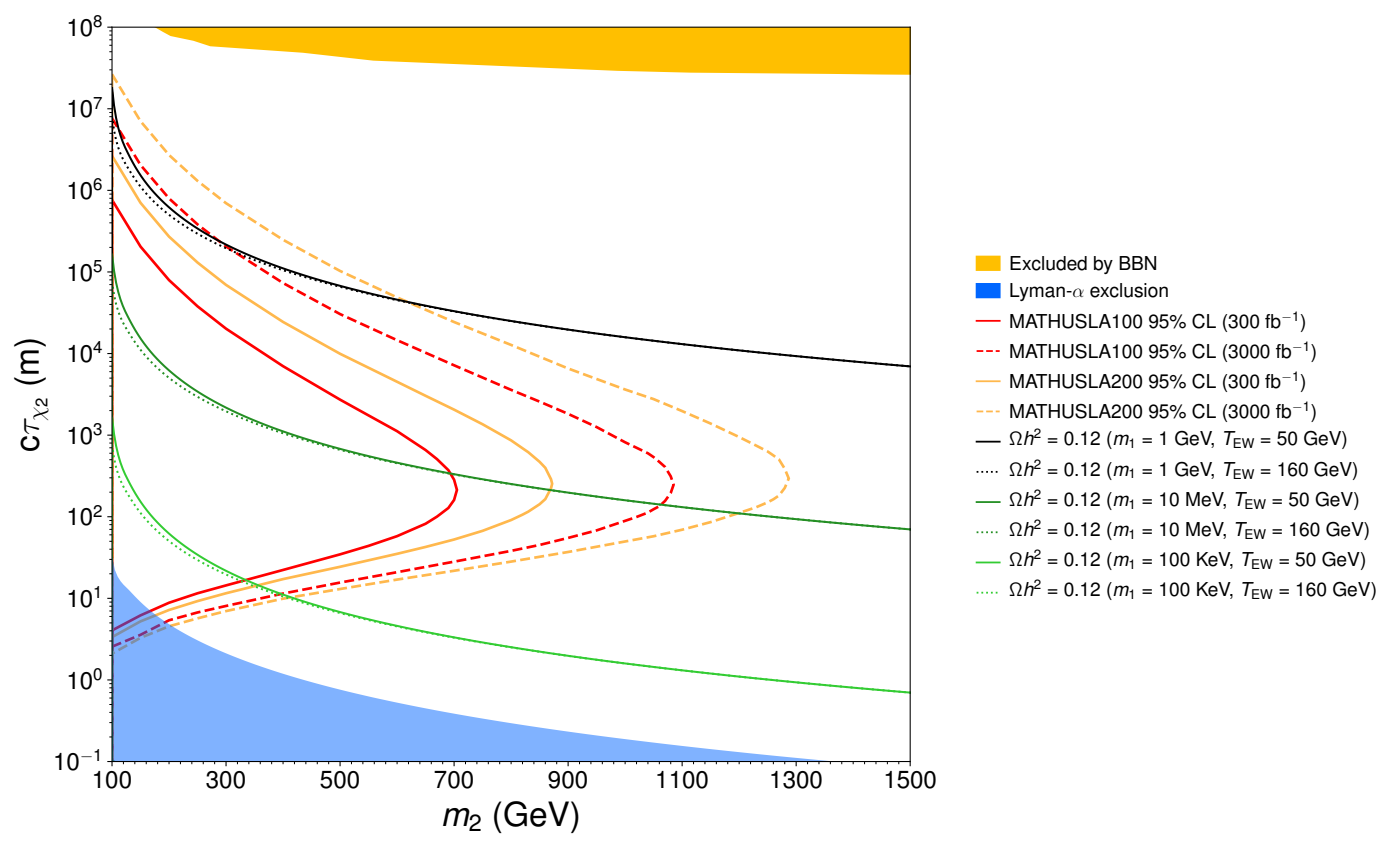

Figure 6. $95 \%$ C.L. exclusion sensitivity (background-free environment assumed, corresponding to $N_{\text {events }}=3$ ) for MATHUSLA200 (orange) and MATHUSLA100 (red) in the $\left(m_{2}, c \tau_{\chi_{2}}\right)$ plane, for $300 \mathrm{fb}^{-1}$ (solid lines) and $3000 \mathrm{fb}^{-1}$ (dashed lines). Lines yielding the observed DM relic density $\Omega_{\mathrm{DM}} h^{2}=0.12$ (obtained with micrOMEGAs5.0) are shown for $m_{1}=1 \mathrm{GeV}$ (black), $10 \mathrm{MeV}$ (dark green), $100 \mathrm{KeV}$ (light green), together with the bounds from BBN (yellow) and Lyman- $\alpha$ (blue).

to the $\chi_{2}$ trajectory, while for $m_{2} \rightarrow m_{h}+m_{1}$ addressing the possible modification of our acceptances requires a more detailed event and detector simulation beyond the scope of this work). We also assume perfect MATHUSLA detection efficiency for $\chi_{2}$ decays inside the detector volume (see nevertheless [82] for a discussion on this point).

The number of expected signal events at MATHUSLA is then given by

$$
N_{\text {events }}=\sigma_{13}^{\mathrm{LHC}} \times \mathcal{L} \times \int P_{\text {decay }}^{\mathrm{MATH}},
$$

where $\mathcal{L}$ is the integrated luminosity and the integral over $P_{\text {decay }}^{\mathrm{MATH}}$ denotes a generic integration over phase-space and decay length for the signal event sample. Considering the MATHUSLA detector as an essentially background-free environment (see [37] for a detailed discussion on this point), the $95 \%$ C.L. exclusion sensitivity corresponds to $N_{\text {events }}=3$.

In figure 6 we show the MATHUSLA100 and MATHUSLA200 95\% C.L. sensitivities in the $\left(m_{2}, c \tau_{\chi_{2}}\right)$ plane for an integrated luminosity $\mathcal{L}=300 \mathrm{fb}^{-1}$ (solid) and $\mathcal{L}=3000 \mathrm{fb}^{-1}$ (dashed). We also show the lines yielding the observed DM relic abundance for $m_{1}=$ $1 \mathrm{GeV}$ (black), $m_{1}=10 \mathrm{MeV}$ (dark green) and $m_{1}=100 \mathrm{KeV}$ (light green) obtained with micrOMEGAs5.0, assuming the temperature of the EW phase transition to be in the range $T_{\mathrm{EW}} \in[50 \mathrm{GeV}, 160 \mathrm{GeV}]$. The results of figure 6 show that MATHUSLA could cover a wide region of the viable freeze-in parameter space between the Lyman- $\alpha$ and BBN constraints, and probe DM masses up to $m_{1} \sim 1-10 \mathrm{GeV}$. In the next section, we compare these sensitivity projections with those of searches by ATLAS and CMS, to investigate the complementarity between MATHUSLA and the existing LHC detectors. 

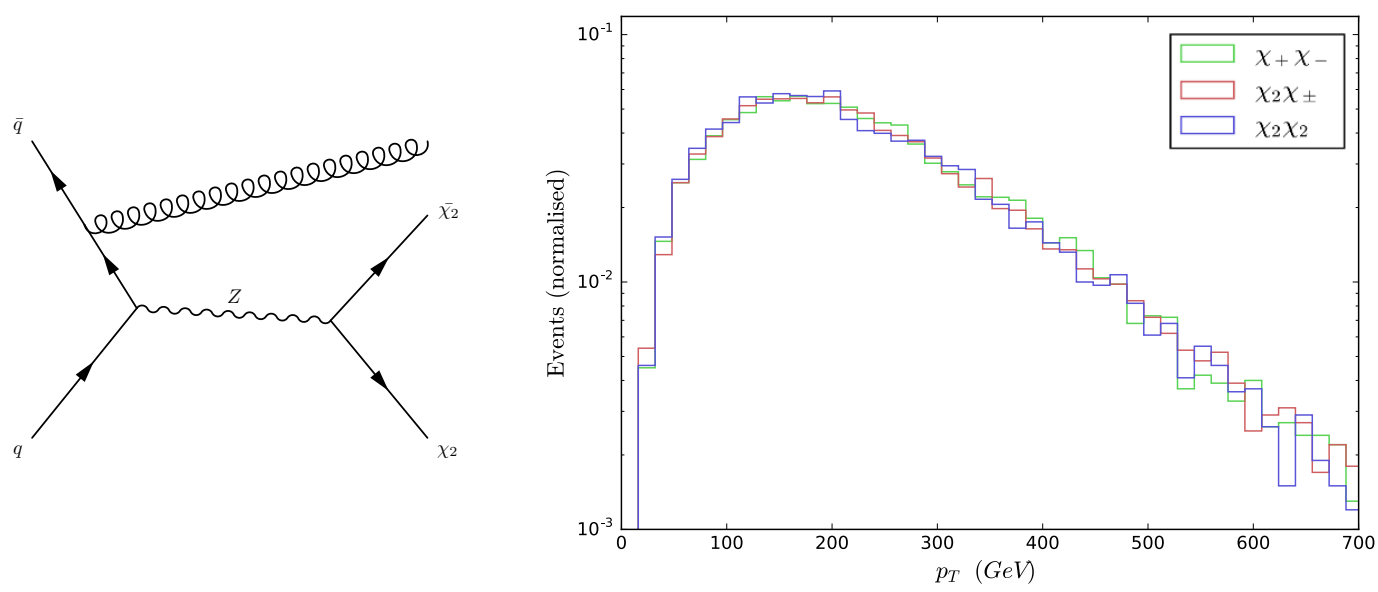

Figure 7. Left: Feynman diagram for the process $p p \rightarrow \bar{\chi}_{2} \chi_{2}+j$. Right: jet $p_{T}$ distribution for $p p \rightarrow \overline{\chi_{2}} \chi_{2}+j$ (blue), $p p \rightarrow \chi_{2} \psi^{+}+j$ (red), $p p \rightarrow \chi_{2} \psi^{-}+j$ (green) and $p p \rightarrow \psi^{+} \psi^{-}+j$ (orange) for $m_{2} \simeq m_{\psi^{ \pm}}=300 \mathrm{GeV}$.

\section{Searches for freeze-in Dark Matter with ATLAS and CMS}

\subsection{Standard Dark Matter searches: $E_{T}^{\text {miss }}$ (mono- $X$ ) signatures}

Due to the long lifetime of $\chi_{2}$, standard "mono- $X$ " searches for DM at the LHC may be sensitive to the freeze-in parameter space when the decay of $\chi_{2}$ happens outside the ATLAS/CMS detector. Here we analyze the sensitivity of such "traditional" DM searches, focusing on the mono-jet signature which generally yields stronger constraints as compared e.g. to mono- $\gamma$. Considering the state $\chi_{2}$ to be invisible (decaying outside the relevant detector volume), jet $+E_{T}^{\text {miss }}$ signatures can be obtained in the current scenario via DrellYan production $p p \rightarrow \overline{\chi_{2}} \chi_{2}, p p \rightarrow \chi_{2} \psi^{ \pm}, p p \rightarrow \psi^{+} \psi^{-}$in association with an initial-stateradiation (ISR) jet, see figure 7 (left). In the latter two processes $\psi^{ \pm}$decays into $\chi_{2}$ and a very soft $\pi^{ \pm}$. The particle $\psi^{ \pm}$will in principle leave a short track in the detector's inner tracker before decaying, but since the decay length of $\psi^{ \pm}$is $\mathcal{O}(1 \mathrm{~cm})$ (see eq. (3.7) and section 6.2), such a short track typically does not affect the mono-jet event selection. ${ }^{20}$

We first focus on the Drell-Yan $p p \rightarrow \overline{\chi_{2}} \chi_{2}$ process with an ISR jet, which is mediated by an off-shell $Z$-boson. This scenario can be reinterpreted as a simplified model for DM (with $\chi_{2}$ as the invisible particle) with a vector mediator $[83,84]$ with $m_{\text {med }}=m_{Z}$, and with the mediator couplings to SM quarks $\left(g_{q}\right)$ and to $\chi_{2}\left(g_{\chi}\right)$ being fixed by the respective gauge quantum numbers of the quarks and $\chi_{2}$. This allows us to use the existing constraints on simplified DM models with vector mediators from the CMS mono-jet analysis at $\sqrt{s}=13 \mathrm{TeV}$ with an integrated luminosity $\mathcal{L}=35.9 \mathrm{fb}^{-1}[6]$. Moreover, since the $p_{T}$ distribution of the ISR jet is similar for all three processes $p p \rightarrow \overline{\chi_{2}} \chi_{2}, p p \rightarrow \chi_{2} \psi^{ \pm}$, $p p \rightarrow \psi^{+} \psi^{-}$, as shown in figure 7 (right), we can to a good approximation rescale the cross

\footnotetext{
${ }^{20}$ The mono-jet analysis features only indirect track vetoes, in the form of well-identified electrons, muons and hadronic $\tau$ leptons, all of which require further activity in the calorimeters or muon stations. That way, the processes $p p \rightarrow \chi_{2} \psi^{ \pm}, p p \rightarrow \psi^{+} \psi^{-}$with an ISR jet may also contribute to the mono-jet signal. We thank Steven Lowette for clarifications on this point.
} 


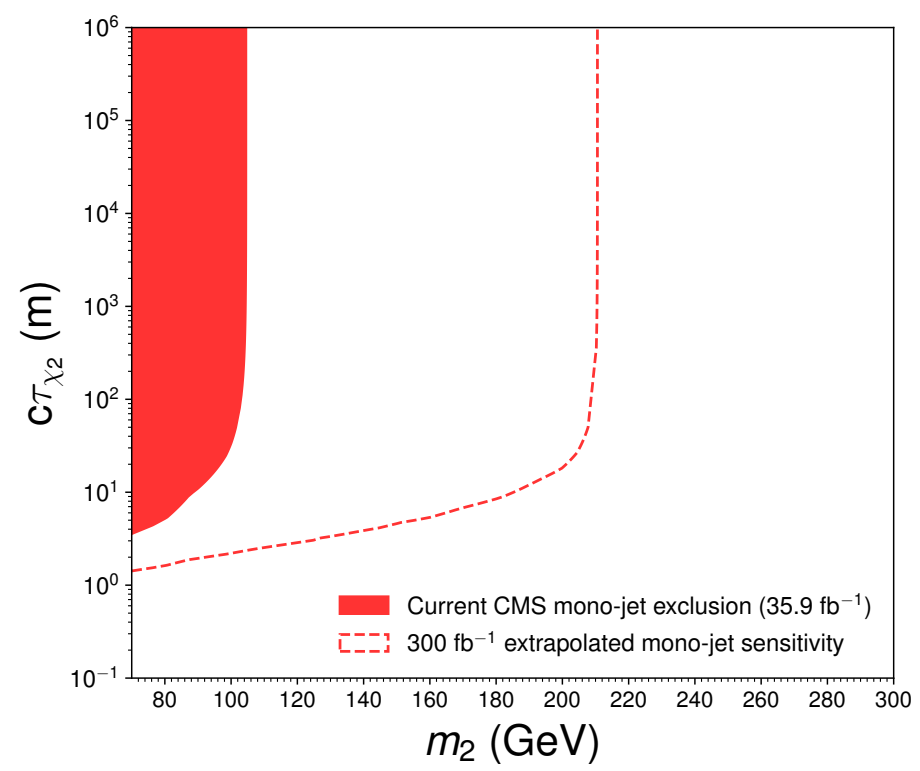

Figure 8. Mono-jet $95 \%$ C.L. exclusion bound in the $\left(m_{2}, \mathrm{c} \tau_{\chi_{2}}\right)$ plane for $35.9 \mathrm{fb}^{-1}$ (solid) and naive extrapolation to $300 \mathrm{fb}^{-1}$ (dashed), see text for details.

section for $p p \rightarrow \overline{\chi_{2}} \chi_{2}+j$ by the total cross section including all three processes (noting also that there is no interference among the different processes).

We then use the publicly provided 95\% C.L. exclusion limit on the mono-jet signal strength $\mu_{\mathrm{CMS}}$ (mono-jet cross section divided by the theoretical cross section for a DM simplified model with a vector mediator and $g_{q}=0.25, g_{\chi}=1$ ) as a function of the DM mass from the CMS analysis [6] for $m_{\text {med }}=m_{Z}$, weighted by the decay probability of $\chi_{2}$ inside the CMS detector. This probability is approximately given by $\exp \left[-\bar{L}_{\mathrm{CMS}} / c \tau_{\chi_{2}}\right]$, with $\bar{L}_{\mathrm{CMS}}$ a mean CMS relevant detector size taken here to be the average hadronic calorimeter radius, $\bar{L}_{\mathrm{CMS}} \sim 2.5$ meters. $^{21}$ The corresponding CMS 95\% C.L. exclusion limit on the present scenario is then given by equating

$$
\mu_{\mathrm{CMS}}\left(m_{2}\right)=\frac{g^{2}}{4 c_{\theta_{W}}^{2}} \frac{\left(g_{L}^{Z}\right)^{2}+\left(g_{R}^{Z}\right)^{2}}{(0.25)^{2}} \times r_{\sigma}\left(m_{2}\right) \times \exp \left[-\bar{L}_{\mathrm{CMS}} / c \tau_{\chi_{2}}\right]
$$

where $\mu_{\mathrm{CMS}}\left(m_{2}\right)$ is the CMS exclusion limit given in [6] for $m_{\mathrm{DM}}=m_{2}$ and $m_{\text {med }}=m_{Z}$, $g_{L}^{Z}$ and $g_{R}^{Z}$ are the couplings of the $Z$-boson to the left and right-handed SM quarks ${ }^{22}$ and $r_{\sigma}\left(m_{2}\right)$ is the ratio between the cross section for $p p \rightarrow \overline{\chi_{2} \chi_{2}}+j$ and the total cross section including all other Drell-Yan processes (which also involve $\psi^{ \pm}$production), which we

\footnotetext{
${ }^{21}$ More accurately, the detector geometry should be convoluted with the rapidity distribution of our signal events and the probability of vetoing a signal event in the mono-jet analysis if the decay happens in different parts of the CMS detector. This is however beyond the scope of this work. The exponential fall-off of the mono-jet sensitivity for small values of $\mathrm{c} \tau_{\chi_{2}}$ shown in figure 8 should then only be taken as approximate.

${ }^{22}$ Here we take the up-type quark couplings, which yield the dominant contribution, to compute the exclusion limit. In practice, both the up and down-type quark contributions should be taken into account, bearing in mind that they are slightly different and this difference can be taken into account by re-weighting the different PDF contributions to the Drell-Yan process as a function of the momentum transfer. We however do not perform such re-weighting here.
} 
compute using Madgraph_aMC@NLO at leading order. From eq. (6.1) we derive the current approximate $95 \%$ C.L. bound $m_{2} \gtrsim 104 \mathrm{GeV}$ in the limit $c \tau_{\chi_{2}} \gg \bar{L}_{\mathrm{CMS}}$, see figure 8 . Using a simple $\sqrt{\mathcal{L}}$ luminosity rescaling, we also provide a naive projection of the limit to $\mathcal{L}=300 \mathrm{fb}^{-1}$ with $\sqrt{s}=13 \mathrm{TeV}$, shown in figure 8 , yielding $m_{2} \gtrsim 210 \mathrm{GeV}$ in the limit $c \tau_{\chi_{2}} \gg \bar{L}_{\mathrm{CMS}}$. We however stress that since this projection has been derived assuming the SM background uncertainties in the mono-jet search are dominantly statistical, and in reality the mono-jet search is systematics dominated and so the true mono-jet sensitivity for $\mathcal{L}=300 \mathrm{fb}^{-1}$ should be significantly weaker than the above value of $m_{2}$. On the other hand, future improvements in the systematic errors would make our (overly aggressive) limit more realistic.

\subsection{Disappearing track signatures}

Due to the very small mass splitting $\delta m \sim 300 \mathrm{MeV}$ between the states $\psi^{ \pm}$and $\chi_{2}$ (recall eq. (3.5)), the state $\psi^{ \pm}$is relatively long-lived, with its decay length in the range $c \tau_{\psi^{ \pm}} \in$ $[0.7,2.1] \mathrm{cm}$ for $m_{\psi^{ \pm}}$between $90 \mathrm{GeV}$ and $1 \mathrm{TeV}$. The dominant decay $\psi \rightarrow \chi_{2} \pi^{ \pm}$can then lead to a disappearing track signature, due to the softness of the produced pion. Both CMS [85] and ATLAS [86] have performed searches for disappearing tracks with LHC $13 \mathrm{TeV}$ data and an integrated luminosity of $36.1 \mathrm{fb}^{-1}\left(38.4 \mathrm{fb}^{-1}\right)$ in the case of ATLAS (CMS). We note that ATLAS can reconstruct tracks as short as $\sim 12 \mathrm{~cm}$, while for the CMS tracker the minimum reconstructed track length is $\sim 25-30 \mathrm{~cm}$ (see e.g. the discussion in $[33,87])$. As a result, the ATLAS search can constrain smaller lifetimes than the CMS study. In fact, the CMS search [85] only constrains chargino lifetimes $\tau>0.07$ nanoseconds (ns) corresponding to $c \tau>2.1 \mathrm{~cm}$, already at the edge of the maximum value of $c \tau_{\psi^{ \pm}}$ possible in the present scenario. Using the exclusion from the $\tau \in[0.07,0.1] \mathrm{ns}, m_{2} \in$ $[50,150] \mathrm{GeV}$ bin in the CMS analysis [85] (yielding $\sigma \times B<17.60 \mathrm{pb}$ ) and using the chargino production cross sections provided in [80], we derive the tentative current bound $m_{\psi^{ \pm}}>84 \mathrm{GeV}$, which is in fact weaker than existing LEP constraints on the state $\psi^{ \pm}[61$, 62]. In contrast, the ATLAS collaboration recently reinterpreted their analysis [86] in terms of pure Higgsino ${ }^{23}$ production [88], obtaining a present $95 \%$ C.L. exclusion of $m_{\psi^{ \pm}} \gtrsim$ $145 \mathrm{GeV}$. In addition, the ATLAS collaboration has performed a preliminary study of their future sensitivity to a disappearing track signature in the pure Higgsino scenario [89], which would yield a $95 \%$ C.L. sensitivity of $m_{\psi^{ \pm}} \sim 260 \mathrm{GeV}$ at the HL-LHC with $\sqrt{s}=14 \mathrm{TeV}$ and $\mathcal{L}=3000 \mathrm{fb}^{-1}$. We show the corresponding limits in figure 9 .

\subsection{Displaced Vertex (DV) signatures}

Neutral long-lived particles may be searched for via displaced vertex signatures in ATLAS, CMS and $\mathrm{LHCb}$, and a wide variety of analyses already exist for $\mathrm{LHC} \sqrt{s}=7,8$ and $13 \mathrm{TeV}$ (see e.g. [45, 90-96]). Among the various searches which could most effectively probe the scenario explored in this work, we highlight:

\footnotetext{
${ }^{23}$ This essentially corresponds to the present scenario, the only difference occurring for values of $\mathrm{c} \tau_{\chi_{2}} \lesssim 10$ $\mathrm{cm}$, for which the decay $\psi^{ \pm} \rightarrow W^{ \pm} \chi_{1}$ starts being important (see figure 1 (right)), which would make the state $\psi^{ \pm}$shorter-lived and thus result in a weakening of the $95 \%$ C.L. exclusion limit from disappearing track searches.
} 
- CMS/ATLAS searches looking for displaced lepton pairs [90], which would be sensitive to decays $\chi_{2} \rightarrow Z \chi_{1}(Z \rightarrow \ell \ell$ ), and to a lesser extent (due to the much smaller leptonic branching fraction) to $\chi_{2} \rightarrow h \chi_{1}\left(h \rightarrow \ell \nu_{\ell} \ell^{\prime} \nu_{\ell^{\prime}}\right)$.

- ATLAS searches looking for activity in the muon spectrometer [92, 96], which could in principle be sensitive to very large decay lengths $c \tau_{\chi_{2}} \gg 1 \mathrm{~m}$.

- ATLAS searches for displaced vertices $+E_{T}^{\text {miss }}$ [45]. These would take advantage of the large hadronic branching fraction of both $Z$ and $h$, as well as of the missing momentum in the decays $\chi_{2} \rightarrow Z / h \chi_{1}$.

In this work, we concentrate on the latter DV $+E_{T}^{\text {miss }}$ search by ATLAS [45] at $\sqrt{s}=13$ $\mathrm{TeV}$ with $32.8 \mathrm{fb}^{-1}$, leaving an exploration of the other two searches highlighted above for future work.

The ATLAS DV $+E_{T}^{\text {miss }}$ search [45] targets events with large missing transverse momentum and one or more displaced vertices with large track multiplicity (thus likely to correspond to hadronic decays). The analysis provides very detailed documentation ${ }^{24}$ for validation and recasting in its auxiliary material [97], and we give details on the analysis selection in appendix B. In order to validate our approach, we have applied the analysis to the model used by ATLAS for interpretation of their results, corresponding to pair-production of long-lived gluinos, with the gluino eventually decaying to a pair of SM quarks and a neutralino. The results of our validation are shown in appendix B, which allow us to perform a recast of the ATLAS search in terms of the Higgs-assisted freeze-in DM scenario explored in this work (an analogous recasting analysis for this scenario has already been performed in [32], with similar results).

As in section 5, we use the Lagrangian implementation of the freeze-in DM model in FeynRules [78]. We simulate Drell-Yan production $p p \rightarrow \chi_{2} \overline{\chi_{2}}$ with the decays of the $\chi_{2} \overline{\chi_{2}}$ pair to $Z \chi_{1} Z \overline{\chi_{1}}, Z \chi_{1} h \overline{\chi_{1}}$ or $h \chi_{1} h \overline{\chi_{1}}$ in Madgraph_aMC@NLO [79]. Since we find the kinematics of $\chi_{2} \overline{\chi_{2}}, \chi_{2} \psi^{ \pm}$, and $\psi^{+} \psi^{-}$production to be very similar (both without and with the production of an extra hard jet, cf. figure 7), we normalize our signal cross section to the sum of respective LHC $\sqrt{s}=13$ charged/neutral Higgsino production cross sections at NLO+NLL obtained from Resummino-2.0.1 [46, 47]. After parton level event generation (we use the TIME_OF_FLIGHT option within Madgraph_aMC@NLO to introduce a non-zero displacement in the decay of $\chi_{2}$ ), our events are passed to Pythia8 [98] which decays the $Z$ and $h$ bosons, and performs showering and hadronisation. Finally we cluster jets using the Fast Jet [99] implementation within Delphes 3 [100]. Further details of our analysis are given in appendix B.

The derived LHC $13 \mathrm{TeV}$ 95\% C.L. limits from the ATLAS DV $+E_{T}^{\text {miss }}$ search with $32.8 \mathrm{fb}^{-1}$ on the $\left(m_{2}, c \tau_{\chi_{2}}\right)$ parameter space of our present scenario are shown in figure 9 , corresponding to a number of signal events $N_{\text {events }}=3$ (since the expected number of

\footnotetext{
${ }^{24}$ We note that CMS analyses searching for a events with an electron and a muon with large impact parameters [95] are very well-documented and sensitive to the present scenario via decays $\chi_{2} \rightarrow h \chi_{1}$ $\left(h \rightarrow e \nu_{e} \mu \nu_{\mu}\right)$. The very small Higgs leptonic branching fraction $(\sim 0.4 \%)$ results in a much weaker sensitivity w.r.t. DV $+E_{T}^{\text {miss }}$ searches (see e.g. [94]).
} 


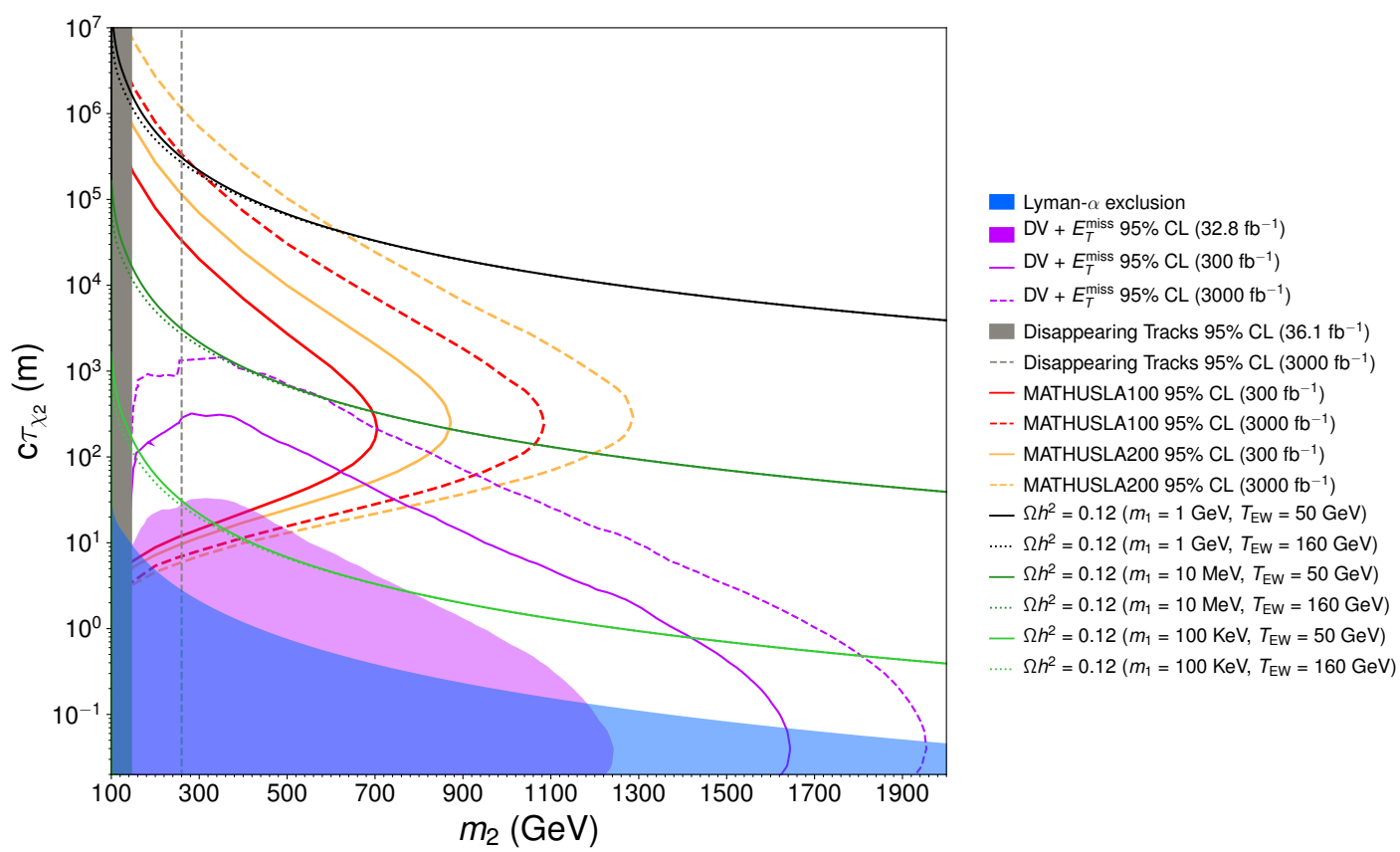

Figure 9. $13 \mathrm{TeV}$ ATLAS DV $+E_{T}^{\text {miss }}$ search $95 \%$ C.L. exclusion limits with $32.8 \mathrm{fb}^{-1}$ (purple region) in the $\left(m_{2}, c \tau_{\chi_{2}}\right)$ plane, together with its extrapolation to $300 \mathrm{fb}^{-1}$ (solid purple line) and $3000 \mathrm{fb}^{-1}$ (dashed purple line). Also shown are the present 95\% C.L. exclusion limits from disappearing track searches (grey region) from section 6.2 and the projection for HL-LHC (vertical dashed grey line), as well as the MATHUSLA100 (red) and MATHUSLA200 (orange) projected $95 \%$ C.L. sensitivities (background-free environment assumed, corresponding to $N_{\text {events }}=3$ ) for $300 \mathrm{fb}^{-1}$ (solid line) and $3000 \mathrm{fb}^{-1}$ (dashed line). Lines yielding the observed DM relic density $\Omega_{\mathrm{DM}} h^{2}=0.12$ (obtained with micrOMEGAs5.0) are shown for $m_{1}=1 \mathrm{GeV}$ (black), $10 \mathrm{MeV}$ (dark green), $100 \mathrm{KeV}$ (light green), together with the bounds from Lyman- $\alpha$ (blue).

background events in the signal region is $0.02_{-0.01}^{+0.02}$, the current search may be considered as background-free). We also show in figure 9 the $95 \%$ C.L. sensitivity projection for the $\mathrm{DV}+E_{T}^{\text {miss }}$ search with $300 \mathrm{fb}^{-1}$ and $3000 \mathrm{fb}^{-1}$. In the former case, the search can still be regarded as background-free and we use $N_{\text {events }}=3$, whereas in the latter we expect $\sim 2$ background events, and the corresponding required number of signal events is found via the $C L s$ method [101] to be $N_{\text {events }}=6$. Finally, we also include in figure 9 the present and projected $95 \%$ C.L. bounds from disappearing track searches (see section 6.2) and the projected $95 \%$ C.L. sensitivity from MATHUSLA100 and MATHUSLA200 (see section 5) to highlight the complementarity among the various searches. While for decay legths $c \tau_{\chi_{2}} \lesssim 100 \mathrm{~m}$ the LHC DV $+E_{T}^{\text {miss }}$ search yields the most sensitive probe of these scenario, for larger decay lengths MATHUSLA provides a major increase in sensitivity w.r.t. the main LHC detectors.

\section{Freeze-in at a $\sqrt{s}=100 \mathrm{TeV}$ collider with a forward detector}

As emphasized in the original MATHUSLA proposal [35], a forward detector at a future proton-proton collider could significantly surpass the capabilities of the MATHUSLA sur- 


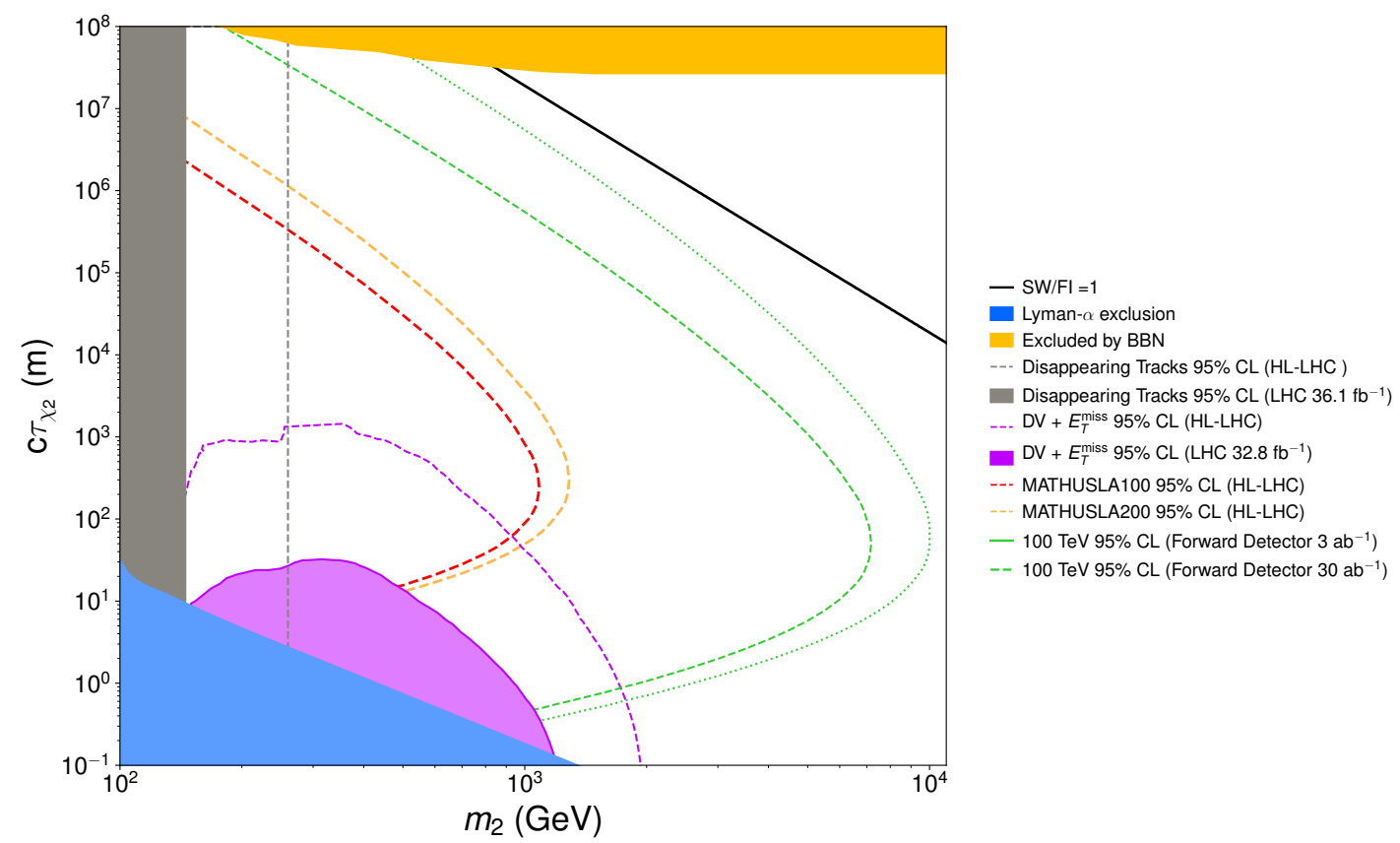

Figure 10. FCC- $h$ h forward detector $95 \%$ C.L. sensitivity projections $\left(N_{\text {events }}=3\right)$ for $\mathcal{L}=3$ $\mathrm{ab}^{-1}$ (dashed-green line) and $\mathcal{L}=30 \mathrm{ab}^{-1}$ (dotted-green line). Shown for comparison are the MATHUSLA100 (dashed-red line) and MATHUSLA200 (dashed-orange line) sensitivity projections (background-free environment assumed, corresponding to $N_{\text {events }}=3$ ) with $\mathcal{L}=3$ ab $^{-1}$ from section 5 , the present $13 \mathrm{TeV}$ ATLAS DV $+E_{T}^{\text {miss }}$ search exclusion limits (purple region) and the extrapolation to HL-LHC (dashed-purple line) from section 6.3, as well as the present exclusion limits from disappearing track searches (grey region) and their extrapolation to HL-LHC (vertical dashed-grey line) from section 6.2. Also shown are the bounds from Lyman- $\alpha$ (blue region) and BBN (yellow region), and the boundary of the super-WIMP parameter-space region (solid-black line), given by $\mathrm{SW} / \mathrm{FI}=1$ (recall figure 4 and the discussion in section 3.3).

face detector proposal to search for very long-lived particles. In this section we analyze the sensitivity to the Higgs-mediated freeze-in DM scenario explored in this work of a $\sqrt{s}=100 \mathrm{TeV}$ hadron collider (from now on referred to as $F C C$ - $h$ h) and a forward LLP detector with volume given by $z \in[20,40] \mathrm{m}, \rho=\sqrt{x^{2}+y^{2}} \in[5,30] \mathrm{m}$ as measured from the $F C C$-hh interaction point.

We simulate Drell-Yan production $p p \rightarrow \chi_{2} \overline{\chi_{2}}, p p \rightarrow \overline{\chi_{2}} \psi^{-}, p p \rightarrow \chi_{2} \psi^{+}, p p \rightarrow \psi^{+} \psi^{-}$ at $\sqrt{s}=100 \mathrm{TeV}$ in Madgraph_aMC@NLO, normalizing the respective cross sections to the corresponding NLO+NLL pure Higgsino production cross sections computed with Resummino-2.0.1, and shown in appendix C. The decays of the charged states into $\chi_{2}$ and $\overline{\chi_{2}}$ are again considered to yield neutral states with approximately identical kinematics to that of the decaying charged states. We follow the same procedure used in section 5 for MATHUSLA to estimate the event yield at the forward detector, computing from simulation the number of signal events $N_{\text {events }}$ from the probability for a particle $\chi_{2}$ to decay within the forward detector volume (we assume perfect detector performance as well as a background-free environment), given by (5.3). The forward detector geometric acceptance 
for our signal is found from simulation to be $\epsilon_{\text {geometric }} \sim 0.5$, which is roughly ten times larger than the one of MATHUSLA due to the rather forward nature of our Drell-Yan events for $\sqrt{s}=100 \mathrm{TeV}$ proton-proton collisions.

In figure 10 we show our FCC-hh forward detector 95\% C.L. sensitivity projections, given by $N_{\text {events }}=3$, for two choices of $F C C$ - $h$ h integrated luminosity, $\mathcal{L}=3 \mathrm{ab}^{-1}$ and $\mathcal{L}=30 \mathrm{ab}^{-1}$. The mass reach shows a dramatic increase w.r.t. MATHUSLA and HLLHC, ${ }^{25}$ being able to probe LLPs with $c \tau_{\chi_{2}} \sim 50 \mathrm{~m}$ up to $m_{2} \sim 10 \mathrm{TeV}$. figure 10 also shows that the forward detector would be able to probe lifetimes up to the BBN bound for $m_{2} \lesssim 600 \mathrm{GeV}$, and approach the boundary of the super-WIMP parameter space region (solid black line in figure 10).

\section{Conclusions}

Freeze-in constitutes a well-motivated and appealing mechanism for DM production in the early Universe, yet challenging to probe experimentally given the feeble interactions between DM and the visible sector. In this sense, collider searches for LLPs have been recently regarded as possible probes of DM freeze-in production via the decay of a parent particle which is accessible at colliders. In this work we have studied the prospects of probing DM freeze-in from the decay of neutral parent particles which belong to the thermal bath, through LLP signatures. Using as a case-study a model featuring Higgs-associated LLP decays, we have obtained the experimental sensitivity of the proposed MATHUSLA surface detector and ATLAS/CMS at HL-LHC to the cosmologically relevant freeze-in DM parameter space. Our results show the high degree of complementarity between MATHUSLA and the main LHC detectors, together being able to probe DM masses from the Lyman- $\alpha$ bound (of a few $\mathrm{keV}$ ) up to masses of few $\mathrm{GeV}$, and neutral parent particle masses up to $\sim 2 \mathrm{TeV}$. Besides, for parent particle masses of $\mathcal{O}(100) \mathrm{GeV}$, MATHUSLA could probe lifetimes close to the BBN limit.

We have also analyzed the improvement in sensitivity that would come from a forward LLP detector within a future $100 \mathrm{TeV}$ hadron collider $(F C C-h h)$, finding that such an improvement would be quite dramatic and would allow to probe parent LLPs with masses up to $10 \mathrm{TeV}$ for $\mathrm{DM}$ masses in the $\mathrm{MeV}$ range, as well as reach the BBN limit for parent LLP masses below $600 \mathrm{GeV}$.

In addition to the above results, we have discussed in some detail several technical aspects of freeze-in DM scenarios: the first aspect, being particular to our Higgs-assisted freeze-in DM model, concerns the EW phase transition, which switches-on some DM production processes when it takes place and plays a key role in regularising certain $2 \rightarrow 2$ scattering processes which would otherwise make DM production UV-sensitive. At the same time, our calculation of the DM production prior to the EW phase transition includes

\footnotetext{
${ }^{25}$ Obviously, a DV $+E_{T}^{\text {miss }}$ search at $F C C$ - $h$ would significantly increase the mass reach of the corresponding search at HL-LHC. Still, already for $c \tau_{\chi_{2}} \gtrsim 1 \mathrm{~m}$ the forward detector is expected to be more sensitive than the main $F C C$ - $h$ detector(s), as the former will have much less background and the geometric acceptances of both are comparable (as opposed to HL-LHC vs MATHUSLA, case in which the smaller geometric acceptance $\epsilon_{\text {geometric }}$ of MATHUSLA results in MATHUSLA being more sensitive than the LHC $\mathrm{DV}+E_{T}^{\mathrm{miss}}$ search only for $\left.c \tau_{\chi_{2}} \gtrsim 100 \mathrm{~m}\right)$.
} 
the corrections due to the thermal mass of the Higgs doublet and the parent particle. The inclusion of thermal masses in DM relic density computations is a rather generic issue that has so far been disregarded for freeze-in via decay scenarios ${ }^{26}$ and can have a significant quantitative impact on the results. The other technical aspects analyzed also apply more generally to models of freeze-in DM production from decay, and correspond to the impact of $2 \rightarrow 2$ scattering processes on the DM relic abundance and the interplay between freeze-in and super-WIMP Dark Matter production mechanisms.

\section{Acknowledgments}

We would like to thank Thomas Konstandin, Alberto Mariotti, Laura Lopez-Honorez, Felix Kahlhoefer, Saniya Heeba, Steven Lowette, Susanne Westhoff and Manimala Chakrabourti for useful discussions. We also thank Benjamin Fuks for pointing us to the use of Resummino. J.M.N. is supported by the Programa Atracción de Talento de la Comunidad de Madrid via grant 2017-T1/TIC-5202. B.Z. acknowledges support from the Programa Atraccion de Talento de la Comunidad de Madrid under grant n. 2017-T2/TIC-5455. P.T. acknowledges support from the Deutsche Forschungsgemeinschaft (DFG) through the Emmy Noether Grant No. KA 4662/1-1 and the Collaborative Research Center TRR 257 "Particle Physics Phenomenology after the Higgs Discovery". J.M.N. and B.Z. acknowledge support from the Spanish MINECO's "Centro de Excelencia Severo Ochoa" Programme via grant SEV-2016-0597. J.M.N. and P.T. were supported by the European Research Council under the European Union's Horizon 2020 program, ERC Grant Agreement 648680 (DARKHORIZONS) during the early stages of this work. J.M.N. thanks the Korean Institute for Advanced Study (KIAS) for hospitality during completion of this work.

\section{A $\quad 1 \rightarrow 2$ vs. $2 \rightarrow 2$ freeze-in processes}

As outlined in section 3.2, whenever there exists a decay $A \rightarrow B_{\mathrm{SM}} \chi$ contributing to freezein DM production, there will be related $2 \rightarrow 2$ scattering processes also contributing to $\mathrm{DM}$ freeze-in at the same order in the freeze-in coupling $y_{\chi}$, where the SM particle $B_{\mathrm{SM}}$ appears in the $t$-channel of the scattering process $X_{\mathrm{SM}}+A \rightarrow Y_{\mathrm{SM}}+\chi$, with both $X_{\mathrm{SM}}$ and $Y_{\mathrm{SM}} \mathrm{SM}$ particles. This is shown in figure 11, with the SM coupling among $X_{\mathrm{SM}}, Y_{\mathrm{SM}}$ and $B_{\mathrm{SM}}$ denoted generically by $g_{\mathrm{SM}}$.

We define a measure of the relative importance of these $2 \rightarrow 2$ scattering processes w.r.t. the $1 \rightarrow 2$ decays $A \rightarrow B_{\mathrm{SM}} \chi$, simply given by the ratio of $\mathrm{DM}$ yields from $1 \rightarrow 2$ and $2 \rightarrow 2$ processes (with thermal corrections neglected in this discussion):

$$
\begin{aligned}
\frac{Y_{2 \rightarrow 2}}{Y_{1 \rightarrow 2}}= & \frac{1}{48 \pi^{3} m_{A}^{2} \Gamma_{A}} \\
& \times \int_{0}^{\infty} x^{3} d x \int_{\left(m_{A}+m_{X}\right)^{2}}^{\infty} \sqrt{s}\left[s-\left(m_{A}+m_{X}\right)^{2}\right] K_{1}\left(\frac{\sqrt{s} x}{m_{A}+m_{X}}\right) \sigma(s) d s \\
= & \frac{1}{32 \pi^{2} m_{A}^{2} \Gamma_{A}} \times \int_{\left(m_{A}+m_{X}\right)^{2}}^{\infty} \frac{\left(m_{A}+m_{X}\right)^{4} \sqrt{s}\left[s-\left(m_{A}+m_{X}\right)^{2}\right]}{s^{2}} \sigma(s) d s
\end{aligned}
$$

\footnotetext{
${ }^{26}$ For freeze-in via scattering, see the very recent studies [17-19]. Note that the model in [18] produces a pair of DM particles from the decay of the parent particle, contrary to our scenario which is the "standard" freeze-in via decay, where only a single DM particle is produced along with a thermal bath particle.
} 

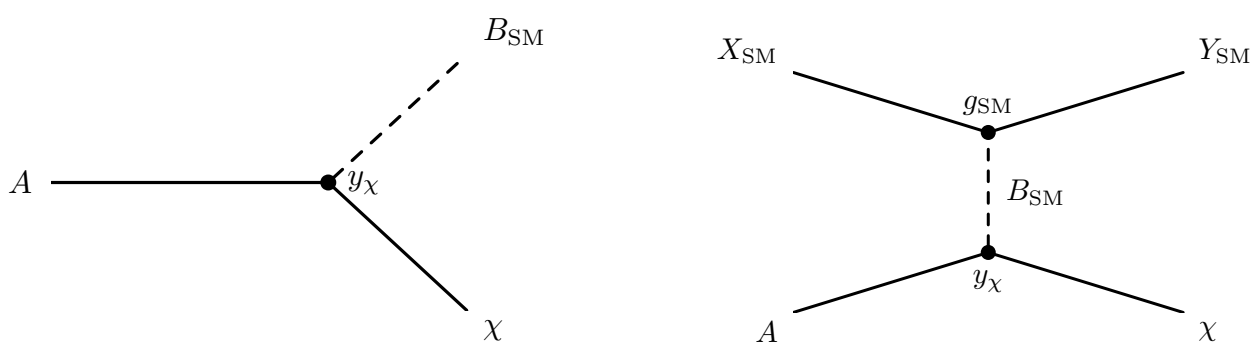

Figure 11. Feynman diagrams for the processes $A \rightarrow B_{\mathrm{SM}} \chi$ (left) and $X_{\mathrm{SM}}+A \rightarrow Y_{\mathrm{SM}}+\chi$ (right).

with $\Gamma_{A}$ the decay width of the process $A \rightarrow B_{\mathrm{SM}} \chi, \sigma(s)$ the cross section for the process $X_{\mathrm{SM}}+A \rightarrow Y_{\mathrm{SM}}+\chi, m_{X}$ and $m_{A}$ the mass of the initial states $A$ and $X_{\mathrm{SM}}$ and $K_{1}$ the first modified Bessel function of the $2^{\text {nd }}$ kind. In the last step of (A.1) we have performed explicitly the integration in $x=m_{A} / T$ (only possible when thermal corrections are neglected).

As discussed above, $\Gamma_{A}, \sigma(s) \propto y_{\chi}^{2}$ and so the ratio (A.1) is independent of $y_{\chi}$ and might turn out to be sizable. In the following, we compute the above ratio in our freeze-in scenario for $B_{\mathrm{SM}}=h$ and $X_{\mathrm{SM}}=Y_{\mathrm{SM}}=q$, a SM quark with Yukawa coupling $y_{q}$ (other possibilities for $X_{\mathrm{SM}}$ and $Y_{\mathrm{SM}}$ such as SM gauge bosons $W, Z$ would yield similar results in this case, being the nature of the $t$-channel mediator the relevant ingredient here). The cross section $\sigma(s)$ has a simple analytic form in the limit $m_{q}, m_{\chi} \rightarrow 0$

$$
\frac{\sigma(s)}{y_{\chi}^{2}}=\frac{y_{q}^{2}}{64 \pi}\left[\frac{\left(s-2 m_{A}^{2}+2 m_{h}^{2}\right)}{\left(s-m_{A}^{2}\right)\left(s-m_{A}^{2}+m_{h}^{2}\right)}+\frac{\left(m_{A}^{2}-2 m_{h}^{2}\right)}{\left(s-m_{A}^{2}\right)^{2}} \log \left(\frac{s-m_{A}^{2}+m_{h}^{2}}{m_{h}^{2}}\right)\right] .
$$

Inserting (A.2) into (A.1) and with $\Gamma_{A}$ given by $\Gamma\left(\chi_{2} \rightarrow h \chi_{1}\right)$ in eq. (3.4), we obtain the ratio $Y_{2 \rightarrow 2} /\left(y_{q}^{2} Y_{1 \rightarrow 2}\right)$ as a function of $m_{A}$, shown in figure 12 . We nevertheless note that for $q$ being the top quark, which corresponds to the leading $2 \rightarrow 2$ scattering process (since $\sigma(s) \propto y_{t}^{2}$ in that case), the top mass $m_{t}$ cannot be neglected. In this case we obtain the cross section $\sigma(s)$ numerically and show the ratio $Y_{2 \rightarrow 2} /\left(y_{t}^{2} Y_{1 \rightarrow 2}\right)$ as a function of $m_{A}$ in figure 12, keeping for simplicity $m_{\chi} \rightarrow 0$. It becomes clear that the decay processes are largely dominant except in the limit $m_{A} \rightarrow m_{h}$, and for $m_{A}<m_{h}$ when 2-body decays are forbidden and the ratio (A.1) should instead be understood as $Y_{2 \rightarrow 2} / Y_{1 \rightarrow 3}$ (the relevant decays of $A$ are 3-body). In figure 12 we also show the region $m_{A}<m_{h}$ down to $m_{A}=100 \mathrm{GeV}$, where the ratio $Y_{2 \rightarrow 2} /\left(y_{q}^{2} Y_{1 \rightarrow 3}\right)$ is very large and $2 \rightarrow 2$ scatterings clearly dominate freeze-in DM production. We stress that $2 \rightarrow 2$ scattering processes with $X_{\mathrm{SM}}$ and $Y_{\mathrm{SM}}$ being SM states other than quarks (e.g. $X_{\mathrm{SM}}=V_{\mathrm{SM}}$ (a SM gauge boson) and $Y_{\mathrm{SM}}=h$ ) may be more important, yet their contribution is expected to be qualitatively of the same order of magnitude as the former (see e.g. figure 5 of [60] in the context of the Type-I see-saw scenario). A complete, detailed analysis of the impact of $2 \rightarrow 2$ scattering processes in freeze-in scenarios is left for future work.

The case where $B_{\mathrm{SM}}$ is an EW gauge boson nevertheless behaves in a different fashion to the above, and is worth discussing separately. Focusing on $B_{\mathrm{SM}}=Z$ in the EW broken phase (the analysis for $B_{\mathrm{SM}}=W^{ \pm}$is analogous), we note that the cross section for the 


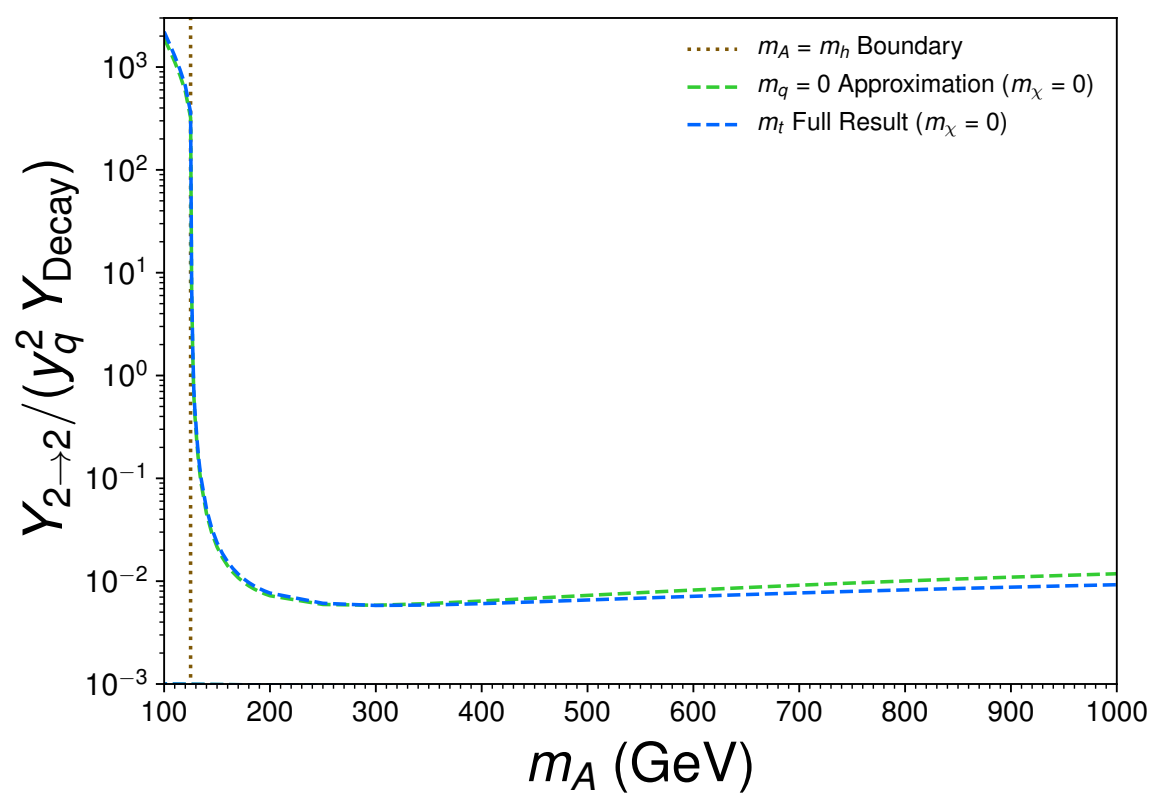

Figure 12. Ratio of decays $\left(1 \rightarrow 2\right.$ for $m_{A}>m_{h}$ and $1 \rightarrow 3$ for $\left.m_{A}<m_{h}\right)$ to $2 \rightarrow 2$ freeze-in DM production processes $Y_{2 \rightarrow 2} /\left(y_{q}^{2} Y_{\text {Decay }}\right)$ as a function of $m_{A}$ and for $m_{\chi}=0$, neglecting $m_{q}$ (green) and including the full $m_{t}$ dependence (blue). The boundary $m_{A}=m_{h}$ (below which the $1 \rightarrow 2$ decay cannot happen) is shown as a brown dotted vertical line.

$2 \rightarrow 2$ scattering process $X_{\mathrm{SM}}+A \rightarrow Y_{\mathrm{SM}}+\chi$ (with $X_{\mathrm{SM}}=Y_{\mathrm{SM}}=q$, a SM quark) mediated by a $t$-channel $Z$ boson is given in the limit $m_{q}, m_{\chi} \rightarrow 0$ by

$$
\begin{aligned}
\frac{\sigma(s)}{y_{\chi}^{2}}= & \frac{m_{Z}^{4}\left(5-4 \cos ^{2} \theta_{W}+8 \cos ^{4} \theta_{W}\right)}{144 \pi m_{A}^{2} v^{2}} \\
& \times\left[\frac{\left[s\left(s+m_{Z}^{2} / 2\right)+\left(s+m_{Z}^{2}\right)\left(m_{Z}^{2}-m_{A}^{2}\right)\right]}{m_{Z}^{2}\left(s-m_{A}^{2}\right)\left(s-m_{A}^{2}+m_{Z}^{2}\right)}\right. \\
& \left.\quad-\frac{s+m_{Z}^{2}-m_{A}^{2} / 2}{\left(s-m_{A}^{2}\right)^{2}} \log \left(\frac{s-m_{A}^{2}+m_{Z}^{2}}{m_{Z}^{2}}\right)\right] .
\end{aligned}
$$

and scales as $m_{A}^{-2}$ (independent of $s$ ) for $s \gg m_{A}^{2}, m_{Z}^{2}$, as opposed to (A.2) which scales as $s^{-2}$ in that limit. Naively, this would lead to a UV divergent result for (A.1) (without considering thermal corrections, which are crucial in this case), signaling a UV dominated freeze-in production mechanism from $2 \rightarrow 2$ scattering processes mediated by a $t$-channel $Z$ boson. We note however that the interaction among $A, \chi$ and the EW gauge bosons vanishes when the EW symmetry is restored above the EW phase transition temperature (recall the discussion in section 3.2), which regulates the integral $\int d s$ in (A.1) and again yields a subdominant contribution from $2 \rightarrow 2$ scattering processes to the freeze-in DM abundance.

Finally, it is worth stressing that in a general scenario (such as ours) with various $1 \rightarrow 2$ and $2 \rightarrow 2$ processes contributing to DM freeze-in, the accurate expression analogous to (A.1) may not be as simple, with different contributions becoming relevant at different temperatures (particularly due to EW symmetry breaking in our scenario). 


\section{B $13 \mathrm{TeV}$ ATLAS DV $+E_{T}^{\text {miss }}$ search recast}

We describe here our recast of the ATLAS search for displaced vertices plus missing transverse energy [45] at $\sqrt{s}=13 \mathrm{TeV}$ with $32.8 \mathrm{fb}^{-1}$. The auxiliary material from the ATLAS search [97] provides efficiencies that can be applied to simulated truth level event samples. The search defines tracks (which will be associated to displaced vertices DV below) as:

- The particle associated to the track is charged and stable.

- The particle has $p_{T}>1 \mathrm{GeV}$.

- The particle has a transverse impact parameter $d_{0}>2 \mathrm{~mm}$.

Displaced vertices are constructed from these tracks. The DV must satisfy:

- $4 \mathrm{~mm}<R<300 \mathrm{~mm}$, where $R=\sqrt{x^{2}+y^{2}}$ is the transverse distance to the interaction point, and $|z|<300 \mathrm{~mm}$.

- The DV must have 5 or more tracks (as defined above) associated to it.

- The DV mass $m_{\mathrm{DV}}$ must be at least $10 \mathrm{GeV}$, calculated from the three-momenta of the tracks (as defined above) associated to it and assuming a charged pion mass for each of the tracks.

Finally, the whole event is required to have:

- $E_{T}^{\text {miss }}>200 \mathrm{GeV}$.

- $75 \%$ of the events should have at least one jet ${ }^{27}$ with $p_{T}>70 \mathrm{GeV}$ or at least two jets with $p_{T}>25 \mathrm{GeV}$.

- At least one displaced vertex (as defined above).

After our signal event simulation with Madgraph_aMC@NLO [79] and Pythia8 [98], followed by jet-clustering using the Fast Jet [99] implementation within Delphes 3 [100], we perform the track, vertex, and event selection using the ROOT [102] output of Delphes 3. For those DV in events that pass all the cuts, we apply the vertex-level and event-level efficiencies provided within the auxiliary material of the ATLAS search, obtaining overall event selection efficiencies for $Z Z+E_{T}^{\text {miss }}, Z h+E_{T}^{\text {miss }}$ and $h h+E_{T}^{\text {miss }}$ signal decay channels, respectively. These overall efficiencies for the three separate channels are a function of the $\chi_{2}$ mass and lifetime, and we therefore generate events for the three different different decay channels and different $\chi_{2}$ masses and decay lengths ${ }^{28} c \tau_{\chi_{2}}$.

\footnotetext{
${ }^{27}$ The ATLAS analysis requires trackless jets, where a trackless jet is one where the scalar sum of the charged particle $p_{T}$ is less than $5 \mathrm{GeV}$ for those particles with small impact parameter with respect to the primary vertex. However, since the small impact parameter is not defined by ATLAS, we are unable to impose the trackless requirement in our recast.

${ }^{28} \mathrm{In}$ practice, we have used a fixed $c \tau_{\chi_{2}}=0.1 \mathrm{~m}$ for event generation, noting that since each displacement variable is proportional to $c \tau_{\chi_{2}}$, we can trivially rescale the position space coordinates of each particle to different values of $c \tau_{\chi_{2}}$, before applying the cuts listed above.
} 


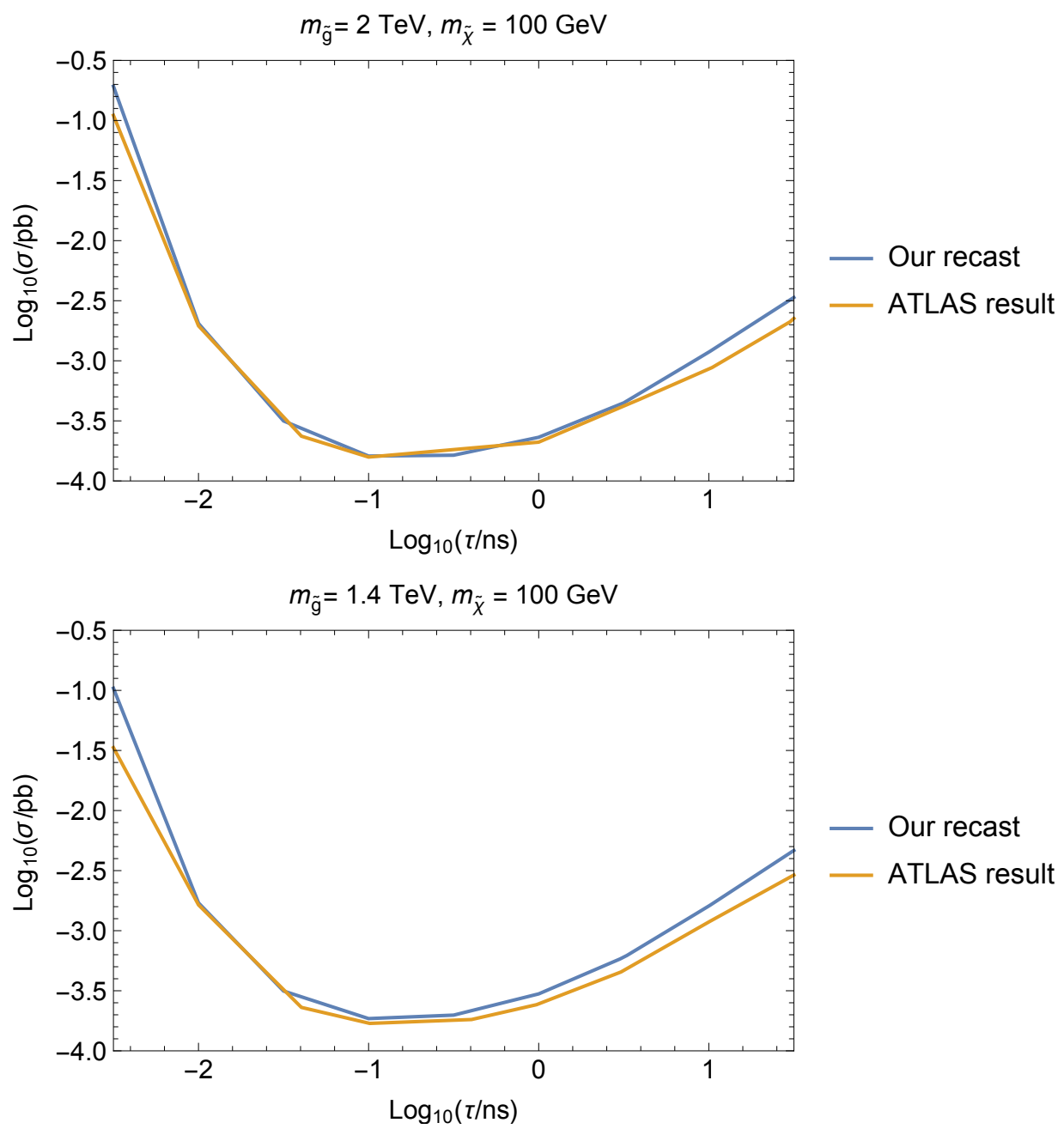

Figure 13. Excluded cross sections at $95 \%$ C.L. as a function of the gluino lifetime, for a long lived gluino decaying to a quark pair and a neutralino, compared to the published result from ATLAS [45]. The gluino mass is fixed to $2 \mathrm{TeV}$ (top) or $1.4 \mathrm{TeV}$ (bottom). The neutralino mass is fixed to $100 \mathrm{GeV}$.

In order to validate the above analysis, we have applied our recast to a long-lived gluino model, where the gluino decays to a quark pair and a neutralino. This is the model used by ATLAS to interpret the results from their DV $+E_{T}^{\text {miss }}$ search. We show our derived 95\% C.L. exclusion limits, as compared to those shown by ATLAS [45], in figure 13 as a function of the gluino lifetime and in figure 14 as a function of the neutralino mass. We find overall good agreement, except in the compressed region (neutralino mass approaching the gluino mass) where our derived limits are stronger than those of ATLAS, signaling that our recasting procedure is less trustworthy for a compressed scenario. In our freeze-in DM model, this happens when the mass of $\chi_{2}$ approaches the SM Higgs mass (the DM candidate $\chi_{1}$ is in general approximately massless on LHC scales), such that our derived limits in that region will be stronger than the actual, would-be ATLAS limits. 


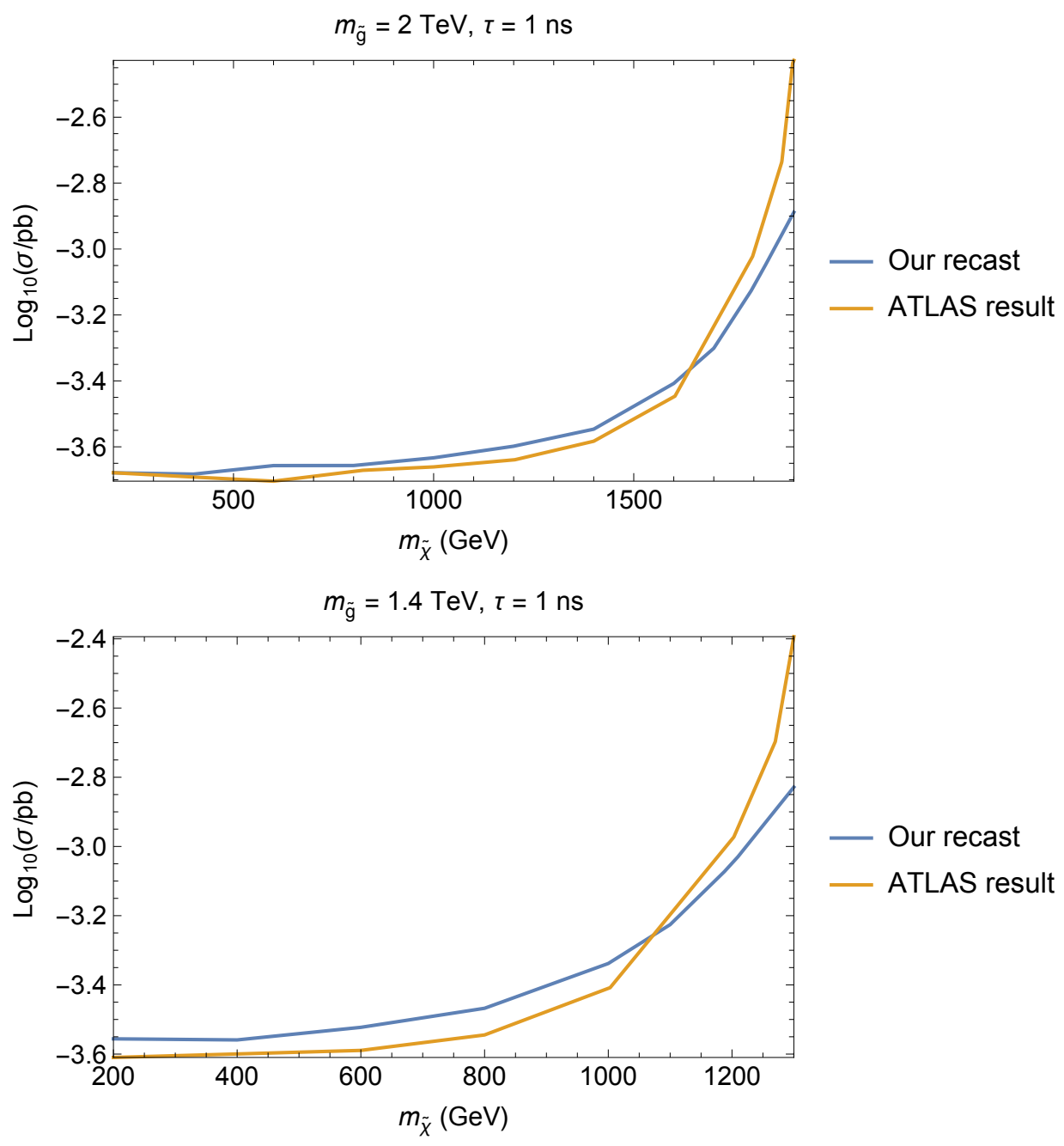

Figure 14. Excluded cross sections at $95 \%$ C.L. as a function of the neutralino mass, for a long lived gluino decaying to a quark pair and a neutralino, compared to the published result from ATLAS [45]. The gluino mass is fixed to $2 \mathrm{TeV}$ (top) or $1.4 \mathrm{TeV}$ (bottom). The gluino lifetime is fixed to $1 \mathrm{~ns}$.

\section{Higgsino pair production cross sections at $\sqrt{s}=13,100 \mathrm{TeV}$}

In this appendix, we show the NLO+NLL pure Higgsino pair production cross sections (assuming decoupled squarks) used in this work, corresponding to $p p \rightarrow \tilde{H}_{1}^{0} \tilde{H}_{2}^{0}, p p \rightarrow$ $\tilde{H}_{1 / 2}^{\tilde{0}} \tilde{H_{1}^{-}}, p p \rightarrow \tilde{H_{1 / 2}^{0}} \tilde{H}_{1}^{+}$and $p p \rightarrow \tilde{H}_{1}^{+} \tilde{H}_{1}^{-}$. These are computed as a function of the Higgsino mass $m_{\tilde{H}} \equiv m_{\tilde{H}_{1}^{ \pm}}=m_{\tilde{H}_{1}^{0}}=m_{\tilde{H}_{2}^{0}}$ from Resummino-2.0.1 [46,47] with the PDF set MSTW2008NLO90CL from LHAPDF [81], both at $\sqrt{s}=13 \mathrm{TeV}$ and $\sqrt{s}=100 \mathrm{TeV}$, and shown in figure 15. The $\sqrt{s}=100 \mathrm{TeV}$ cross sections are found to be in qualitative agreement with the LO pure Higgsino pair production cross sections computed in [103].

Open Access. This article is distributed under the terms of the Creative Commons Attribution License (CC-BY 4.0), which permits any use, distribution and reproduction in any medium, provided the original author(s) and source are credited. 


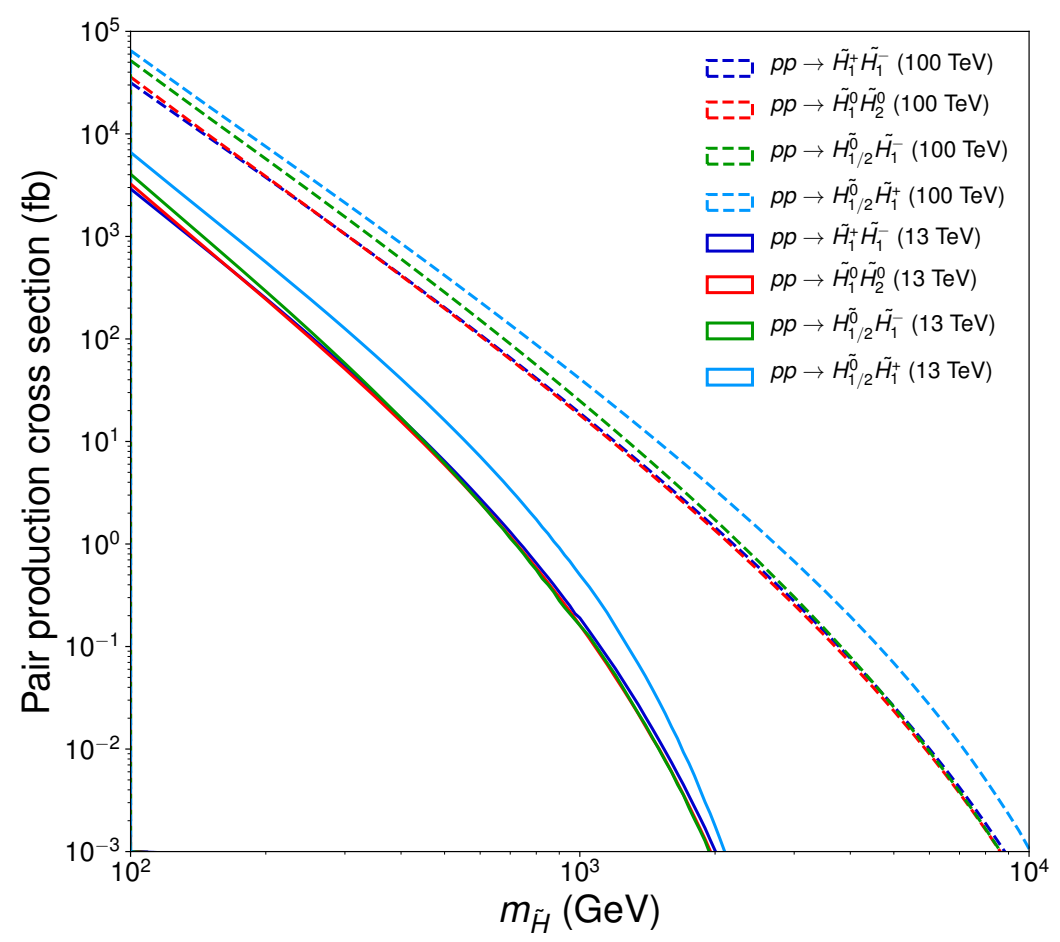

Figure 15. $13 \mathrm{TeV}$ (solid) and $100 \mathrm{TeV}$ (dashed) NLO+NLL pure Higgsino pair production cross sections (assuming decoupled squarks) as a function of the Higgsino mass $m_{\tilde{H}}$ obtained with Resummino-2.0.1 with the PDF set MSTW2008NLO90CL. The processes shown are $p p \rightarrow \tilde{H}_{1}^{0} \tilde{H}_{2}^{0}$ (red), $p p \rightarrow \tilde{H}_{1 / 2}^{\tilde{0}} \tilde{H}_{1}^{-}$(green), $p p \rightarrow \tilde{H}_{1 / 2}^{\tilde{0}} \tilde{H}_{1}^{+}$(light blue) and $p p \rightarrow \tilde{H}_{1}^{+} \tilde{H}_{1}^{-}$(dark blue).

\section{References}

[1] PandaX-II collaboration, Dark matter results from 54-ton-day exposure of PandaX-II experiment, Phys. Rev. Lett. 119 (2017) 181302 [arXiv:1708.06917] [INSPIRE].

[2] XENON collaboration, Dark matter search results from a one ton-year exposure of XENON1T, Phys. Rev. Lett. 121 (2018) 111302 [arXiv:1805.12562] [INSPIRE].

[3] H.E.S.S. collaboration, Search for dark matter annihilations towards the inner Galactic halo from 10 years of observations with H.E.S.S., Phys. Rev. Lett. 117 (2016) 111301 [arXiv: 1607.08142] [INSPIRE].

[4] MAGIC, FERMI-LAT collaboration, Limits to dark matter annihilation cross-section from a combined analysis of MAGIC and Fermi-LAT observations of dwarf satellite galaxies, JCAP 02 (2016) 039 [arXiv: 1601.06590] [INSPIRE].

[5] ATLAS collaboration, Search for dark matter and other new phenomena in events with an energetic jet and large missing transverse momentum using the ATLAS detector, JHEP 01 (2018) 126 [arXiv:1711.03301] [INSPIRE].

[6] CMS collaboration, Search for new physics in final states with an energetic jet or a hadronically decaying $W$ or $Z$ boson and transverse momentum imbalance at $\sqrt{s}=13 \mathrm{TeV}$, Phys. Rev. D 97 (2018) 092005 [arXiv:1712.02345] [INSPIRE]. 
[7] J. McDonald, Thermally generated gauge singlet scalars as selfinteracting dark matter, Phys. Rev. Lett. 88 (2002) 091304 [hep-ph/0106249] [INSPIRE].

[8] L.J. Hall, K. Jedamzik, J. March-Russell and S.M. West, Freeze-in production of FIMP dark matter, JHEP 03 (2010) 080 [arXiv:0911.1120] [INSPIRE].

[9] N. Bernal, M. Heikinheimo, T. Tenkanen, K. Tuominen and V. Vaskonen, The dawn of FIMP dark matter: a review of models and constraints, Int. J. Mod. Phys. A 32 (2017) 1730023 [arXiv: 1706.07442] [INSPIRE].

[10] L. Covi, J.E. Kim and L. Roszkowski, Axinos as cold dark matter, Phys. Rev. Lett. 82 (1999) 4180 [hep-ph/9905212] [INSPIRE].

[11] C. Cheung, G. Elor and L. Hall, Gravitino freeze-in, Phys. Rev. D 84 (2011) 115021 [arXiv:1103.4394] [INSPIRE].

[12] T. Asaka, K. Ishiwata and T. Moroi, Right-handed sneutrino as cold dark matter, Phys. Rev. D 73 (2006) 051301 [hep-ph/0512118] [INSPIRE].

[13] K.-H. Tsao, FIMP dark matter freeze-in gauge mediation and hidden sector, J. Phys. G 45 (2018) 075001 [arXiv: 1710.06572] [inSPIRE].

[14] A. Goudelis, K.A. Mohan and D. Sengupta, Clockworking FIMPs, JHEP 10 (2018) 014 [arXiv: 1807.06642] [INSPIRE].

[15] M. Garny and J. Heisig, Interplay of super-WIMP and freeze-in production of dark matter, Phys. Rev. D 98 (2018) 095031 [arXiv: 1809.10135] [INSPIRE].

[16] S. Heeba, F. Kahlhoefer and P. Stöcker, Freeze-in production of decaying dark matter in five steps, JCAP 11 (2018) 048 [arXiv: 1809.04849] [INSPIRE].

[17] S. Heeba and F. Kahlhoefer, Probing the freeze-in mechanism in dark matter models with $U(1)^{\prime}$ gauge extensions, arXiv:1908.09834 [INSPIRE].

[18] L. Darmé, A. Hryczuk, D. Karamitros and L. Roszkowski, Forbidden frozen-in dark matter, JHEP 11 (2019) 159 [arXiv: 1908.05685] [INSPIRE].

[19] T. Hambye, M.H.G. Tytgat, J. Vandecasteele and L. Vanderheyden, Dark matter from dark photons: a taxonomy of dark matter production, Phys. Rev. D 100 (2019) 095018 [arXiv: 1908.09864] [INSPIRE].

[20] Y. Mambrini, K.A. Olive, J. Quevillon and B. Zaldivar, Gauge coupling unification and nonequilibrium thermal dark matter, Phys. Rev. Lett. 110 (2013) 241306 [arXiv: 1302.4438] [INSPIRE].

[21] S. Koren and R. McGehee, Freeze-twin dark matter, arXiv:1908.03559 [INSPIRE].

[22] F. Elahi, C. Kolda and J. Unwin, Ultraviolet freeze-in, JHEP 03 (2015) 048 [arXiv:1410.6157] [INSPIRE].

[23] F. Kahlhoefer, On the LHC sensitivity for non-thermalised hidden sectors, Phys. Lett. B 779 (2018) 388 [arXiv:1801.07621] [INSPIRE].

[24] N. Bernal et al., Production regimes for self-interacting dark matter, JCAP 03 (2016) 018 [arXiv: 1510.08063] [INSPIRE].

[25] T. Hambye, M.H.G. Tytgat, J. Vandecasteele and L. Vanderheyden, Dark matter direct detection is testing freeze-in, Phys. Rev. D 98 (2018) 075017 [arXiv:1807.05022] [INSPIRE]. 
[26] M. Heikinheimo, T. Tenkanen and K. Tuominen, Prospects for indirect detection of frozen-in dark matter, Phys. Rev. D 97 (2018) 063002 [arXiv:1801.03089] [INSPIRE].

[27] J. Alimena et al., Searching for long-lived particles beyond the standard model at the Large Hadron Collider, arXiv: 1903.04497 [INSPIRE].

[28] D. Curtin et al., Long-lived particles at the energy frontier: the MATHUSLA physics case, Rept. Prog. Phys. 82 (2019) 116201 [arXiv:1806.07396] [inSPIRE].

[29] R.T. Co, F. D'Eramo, L.J. Hall and D. Pappadopulo, Freeze-in dark matter with displaced signatures at colliders, JCAP 12 (2015) 024 [arXiv: 1506.07532] [INSPIRE].

[30] A.G. Hessler, A. Ibarra, E. Molinaro and S. Vogl, Probing the scotogenic FIMP at the LHC, JHEP 01 (2017) 100 [arXiv:1611.09540] [INSPIRE].

[31] A. Ghosh, T. Mondal and B. Mukhopadhyaya, Heavy stable charged tracks as signatures of non-thermal dark matter at the LHC : a study in some non-supersymmetric scenarios, JHEP 12 (2017) 136 [arXiv:1706.06815] [INSPIRE].

[32] L. Calibbi, L. Lopez-Honorez, S. Lowette and A. Mariotti, Singlet-doublet dark matter freeze-in: LHC displaced signatures versus cosmology, JHEP 09 (2018) 037 [arXiv: 1805.04423] [INSPIRE].

[33] G. Bélanger et al., LHC-friendly minimal freeze-in models, JHEP 02 (2019) 186 [arXiv: 1811.05478] [INSPIRE].

[34] A. Filimonova and S. Westhoff, Long live the Higgs portal!, JHEP 02 (2019) 140 [arXiv: 1812.04628] [INSPIRE].

[35] J.P. Chou, D. Curtin and H.J. Lubatti, New detectors to explore the lifetime frontier, Phys. Lett. B 767 (2017) 29 [arXiv: 1606. 06298] [INSPIRE].

[36] D. Curtin and M.E. Peskin, Analysis of long lived particle decays with the MATHUSLA detector, Phys. Rev. D 97 (2018) 015006 [arXiv:1705.06327] [INSPIRE].

[37] MATHUSLA collaboration, A letter of intent for MATHUSLA: a dedicated displaced vertex detector above ATLAS or CMS, arXiv:1811.00927 [INSPIRE].

[38] J.L. Feng, I. Galon, F. Kling and S. Trojanowski, ForwArd Search ExpeRiment at the LHC, Phys. Rev. D 97 (2018) 035001 [arXiv:1708.09389] [InSPIRE].

[39] FASER collaboration, Technical proposal for FASER: ForwArd Search ExpeRiment at the $L H C$, arXiv: 1812.09139 [INSPIRE].

[40] V.V. Gligorov, S. Knapen, M. Papucci and D.J. Robinson, Searching for long-lived particles: a compact detector for exotics at LHCb, Phys. Rev. D 97 (2018) 015023 [arXiv: 1708.09395] [INSPIRE].

[41] V.V. Gligorov et al., Leveraging the ALICE/L3 cavern for long-lived particle searches, Phys. Rev. D 99 (2019) 015023 [arXiv: 1810.03636] [INSPIRE].

[42] L. Covi, H.-B. Kim, J.E. Kim and L. Roszkowski, Axinos as dark matter, JHEP 05 (2001) 033 [hep-ph/0101009] [INSPIRE].

[43] R.T. Co, F. D'Eramo and L.J. Hall, Gravitino or axino dark matter with reheat temperature as high as $10^{16} \mathrm{GeV}$, JHEP 03 (2017) 005 [arXiv: 1611.05028] [INSPIRE].

[44] L. Calibbi, A. Mariotti and P. Tziveloglou, Singlet-doublet model: dark matter searches and LHC constraints, JHEP 10 (2015) 116 [arXiv:1505.03867] [INSPIRE]. 
[45] ATLAS collaboration, Search for long-lived, massive particles in events with displaced vertices and missing transverse momentum in $\sqrt{s}=13$ TeV pp collisions with the ATLAS detector, Phys. Rev. D 97 (2018) 052012 [arXiv: 1710.04901] [INSPIRE].

[46] B. Fuks, M. Klasen, D.R. Lamprea and M. Rothering, Gaugino production in proton-proton collisions at a center-of-mass energy of 8 TeV, JHEP 10 (2012) 081 [arXiv:1207.2159] [INSPIRE].

[47] B. Fuks, M. Klasen, D.R. Lamprea and M. Rothering, Precision predictions for electroweak superpartner production at hadron colliders with Resummino, Eur. Phys. J. C 73 (2013) 2480 [arXiv: 1304.0790] [INSPIRE].

[48] F. D'Eramo, N. Fernandez and S. Profumo, Dark matter freeze-in production in fast-expanding universes, JCAP 02 (2018) 046 [arXiv: 1712.07453] [INSPIRE].

[49] G. Bélanger et al., MicrOMEGAs5.0: freeze-in, Comput. Phys. Commun. 231 (2018) 173 [arXiv: 1801.03509] [INSPIRE].

[50] S.D. Thomas and J.D. Wells, Phenomenology of massive vectorlike doublet leptons, Phys. Rev. Lett. 81 (1998) 34 [hep-ph/9804359] [INSPIRE].

[51] M. Cirelli and A. Strumia, Minimal dark matter: model and results, New J. Phys. 11 (2009) 105005 [arXiv: 0903.3381] [INSPIRE].

[52] M.E. Carrington, The effective potential at finite temperature in the standard model, Phys. Rev. D 45 (1992) 2933 [INSPIRE].

[53] D. Comelli and J.R. Espinosa, Bosonic thermal masses in supersymmetry, Phys. Rev. D 55 (1997) 6253 [hep-ph/9606438] [INSPIRE].

[54] A. Katz and M. Perelstein, Higgs couplings and electroweak phase transition, JHEP 07 (2014) 108 [arXiv: 1401.1827] [INSPIRE].

[55] V.S. Rychkov and A. Strumia, Thermal production of gravitinos, Phys. Rev. D 75 (2007) 075011 [hep-ph/0701104] [INSPIRE].

[56] T. Hambye and D. Teresi, Baryogenesis from L-violating Higgs-doublet decay in the density-matrix formalism, Phys. Rev. D 96 (2017) 015031 [arXiv:1705.00016] [INSPIRE].

[57] M. D'Onofrio, K. Rummukainen and A. Tranberg, Sphaleron rate in the minimal standard model, Phys. Rev. Lett. 113 (2014) 141602 [arXiv:1404.3565] [INSPIRE].

[58] M. D'Onofrio and K. Rummukainen, Standard model cross-over on the lattice, Phys. Rev. D 93 (2016) 025003 [arXiv: 1508.07161] [INSPIRE].

[59] O. Lebedev and T. Toma, Relativistic freeze-in, Phys. Lett. B 798 (2019) 134961 [arXiv: 1908.05491] [INSPIRE].

[60] D. Besak and D. Bödeker, Thermal production of ultrarelativistic right-handed neutrinos: complete leading-order results, JCAP 03 (2012) 029 [arXiv:1202.1288] [INSPIRE].

[61] LEP2 SUSY Working Group, ALEPH, DELPHI, L3 ANd OPAL collaborations, http://lepsusy.web.cern.ch/lepsusy/www/inoslowdmsummer02/charginolowdm_pub.html.

[62] D. Egana-Ugrinovic, M. Low and J.T. Ruderman, Charged fermions below $100 \mathrm{GeV}$, JHEP 05 (2018) 012 [arXiv: 1801.05432] [INSPIRE].

[63] N. Arkani-Hamed, A. Delgado and G.F. Giudice, The well-tempered neutralino, Nucl. Phys. B 741 (2006) 108 [hep-ph/0601041] [INSPIRE]. 
[64] J.L. Feng, A. Rajaraman and F. Takayama, Superweakly interacting massive particles, Phys. Rev. Lett. 91 (2003) 011302 [hep-ph/0302215] [INSPIRE].

[65] A. Goudelis, M. Pospelov and J. Pradler, Light particle solution to the cosmic lithium problem, Phys. Rev. Lett. 116 (2016) 211303 [arXiv:1510.08858] [INSPIRE].

[66] F. Iocco et al., Primordial nucleosynthesis: from precision cosmology to fundamental physics, Phys. Rept. 472 (2009) 1 [arXiv:0809.0631] [INSPIRE].

[67] R.H. Cyburt, B.D. Fields, K.A. Olive and T.-H. Yeh, Big Bang nucleosynthesis: 2015, Rev. Mod. Phys. 88 (2016) 015004 [arXiv:1505.01076] [inSPIRE].

[68] W. Hu and J. Silk, Thermalization constraints and spectral distortions for massive unstable relic particles, Phys. Rev. Lett. 70 (1993) 2661 [INSPIRE].

[69] M. Kawasaki, K. Kohri, T. Moroi and Y. Takaesu, Revisiting Big-Bang nucleosynthesis constraints on long-lived decaying particles, Phys. Rev. D 97 (2018) 023502 [arXiv: 1709.01211] [INSPIRE].

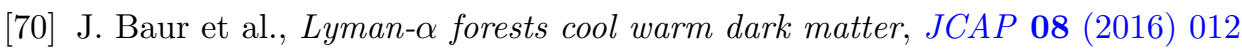
[arXiv: 1512.01981] [INSPIRE].

[71] C. Yèche et al., Constraints on neutrino masses from Lyman-alpha forest power spectrum with BOSS and XQ-100, JCAP 06 (2017) 047 [arXiv: 1702.03314] [INSPIRE].

[72] V. Iršič et al., New Constraints on the free-streaming of warm dark matter from intermediate and small scale Lyman- $\alpha$ forest data, Phys. Rev. D 96 (2017) 023522 [arXiv: 1702.01764] [INSPIRE].

[73] J. Heeck and D. Teresi, Cold keV dark matter from decays and scatterings, Phys. Rev. D 96 (2017) 035018 [arXiv: 1706. 09909] [inSPIRE].

[74] S. Boulebnane, J. Heeck, A. Nguyen and D. Teresi, Cold light dark matter in extended seesaw models, JCAP 04 (2018) 006 [arXiv: 1709.07283] [INSPIRE].

[75] K.J. Bae, A. Kamada, S.P. Liew and K. Yanagi, Light axinos from freeze-in: production processes, phase space distributions and Ly- $\alpha$ forest constraints, JCAP 01 (2018) 054 [arXiv:1707.06418] [INSPIRE].

[76] A. Kamada and K. Yanagi, Constraining FIMP from the structure formation of the Universe: analytic mapping from $m_{W D M}$, JCAP 11 (2019) 029 [arXiv:1907.04558] [INSPIRE].

[77] J. Baur et al., Constraints from Ly- $\alpha$ forests on non-thermal dark matter including resonantly-produced sterile neutrinos, JCAP 12 (2017) 013 [arXiv:1706.03118] [INSPIRE].

[78] A. Alloul et al., FeynRules 2.0 - A complete toolbox for tree-level phenomenology, Comput. Phys. Commun. 185 (2014) 2250 [arXiv:1310.1921] [INSPIRE].

[79] J. Alwall et al., The automated computation of tree-level and next-to-leading order differential cross sections and their matching to parton shower simulations, JHEP $\mathbf{0 7}$ (2014) 079 [arXiv: 1405.0301] [INSPIRE].

[80] LHC SUSY Cross Section Working Group, https://twiki.cern.ch/twiki/bin/view/LHCPhysics/SUSYCrossSections.

[81] A. Buckley et al., LHAPDF6: parton density access in the LHC precision era, Eur. Phys. J. C 75 (2015) 132 [arXiv:1412.7420] [INSPIRE]. 
[82] A. Ibarra, E. Molinaro and S. Vogl, Potential for probing three-body decays of Long-Lived Particles with MATHUSLA, Phys. Lett. B 789 (2019) 127 [arXiv:1809.01683] [InSPIRE].

[83] J. Abdallah et al., Simplified models for dark matter searches at the LHC, Phys. Dark Univ. 9-10 (2015) 8 [arXiv:1506.03116] [INSPIRE].

[84] G. Busoni et al., Recommendations on presenting LHC searches for missing transverse energy signals using simplified s-channel models of dark matter, Phys. Dark Univ. 27 (2020) 100365 [arXiv: 1603.04156] [INSPIRE].

[85] CMS collaboration, Search for disappearing tracks as a signature of new long-lived particles in proton-proton collisions at $\sqrt{s}=13 \mathrm{TeV}$, JHEP 08 (2018) 016 [arXiv:1804.07321] [INSPIRE].

[86] ATLAS collaboration, Search for long-lived charginos based on a disappearing-track signature in pp collisions at $\sqrt{s}=13 \mathrm{TeV}$ with the ATLAS detector, JHEP 06 (2018) 022 [arXiv: 1712.02118] [INSPIRE].

[87] J.A. Evans and J. Shelton, Long-lived staus and displaced leptons at the LHC, JHEP 04 (2016) 056 [arXiv: 1601.01326] [INSPIRE].

[88] ATLAS collaboration, Search for direct pair production of higgsinos by the reinterpretation of the disappearing track analysis with $36.1 \mathrm{fb}^{-1}$ of $\sqrt{\mathrm{s}}=13 \mathrm{TeV}$ data collected with the ATLAS experiment, ATL-PHYS-PUB-2017-019 (2017).

[89] ATLAS collaboration, ATLAS sensitivity to winos and higgsinos with a highly compressed mass spectrum at the HL-LHC, ATL-PHYS-PUB-2018-031 (2018).

[90] CMS collaboration, Search for long-lived particles that decay into final states containing two electrons or two muons in proton-proton collisions at $\sqrt{s}=8$ TeV, Phys. Rev. D 91 (2015) 052012 [arXiv:1411.6977] [INSPIRE].

[91] LHCb collaboration, Search for long-lived particles decaying to jet pairs, Eur. Phys. J. C 75 (2015) 152 [arXiv: 1412.3021] [INSPIRE].

[92] ATLAS collaboration, Search for long-lived, weakly interacting particles that decay to displaced hadronic jets in proton-proton collisions at $\sqrt{s}=8$ TeV with the ATLAS detector, Phys. Rev. D 92 (2015) 012010 [arXiv: 1504.03634] [INSPIRE].

[93] ATLAS collaboration, Search for massive, long-lived particles using multitrack displaced vertices or displaced lepton pairs in pp collisions at $\sqrt{s}=8 \mathrm{TeV}$ with the ATLAS detector, Phys. Rev. D 92 (2015) 072004 [arXiv:1504.05162] [INSPIRE].

[94] Z. Liu and B. Tweedie, The Fate of Long-Lived Superparticles with Hadronic Decays after LHC Run 1, JHEP 06 (2015) 042 [arXiv: 1503.05923] [INSPIRE].

[95] CMS collaboration, Search for displaced leptons $n$ the e- $\mu$ channel, CMS-PAS-EXO-16-022 (2016).

[96] ATLAS collaboration, Search for long-lived particles produced in pp collisions at $\sqrt{s}=13$ TeV that decay into displaced hadronic jets in the ATLAS muon spectrometer, Phys. Rev. D 99 (2019) 052005 [arXiv: 1811.07370] [INSPIRE].

[97] ATLAS collaboration, Search for long-lived, massive particles in events with displaced vertices and missing transverse momentum in $\sqrt{s}=13 \mathrm{TeV}$ pp collisions with the ATLAS detector, auxiliary figures and tables (2017). 
[98] T. Sjöstrand et al., An introduction to PYTHIA 8.2, Comput. Phys. Commun. 191 (2015) 159 [arXiv: 1410.3012] [INSPIRE].

[99] M. Cacciari, G.P. Salam and G. Soyez, FastJet user manual, Eur. Phys. J. C 72 (2012) 1896 [arXiv:1111.6097] [INSPIRE].

[100] DELPHES 3 collaboration, DELPHES 3, A modular framework for fast simulation of a generic collider experiment, JHEP 02 (2014) 057 [arXiv: 1307.6346] [INSPIRE].

[101] A.L. Read, Presentation of search results: the CL technique, J. Phys. G 28 (2002) 2693 [INSPIRE].

[102] I. Antcheva et al., ROOT: A $C++$ framework for petabyte data storage, statistical analysis and visualization, Comput. Phys. Commun. 180 (2009) 2499 [arXiv:1508. 07749] [INSPIRE].

[103] B.S. Acharya, K. Bożek, C. Pongkitivanichkul and K. Sakurai, Prospects for observing charginos and neutralinos at a 100 TeV proton-proton collider, JHEP 02 (2015) 181 [arXiv:1410.1532] [INSPIRE]. 